\title{
CO line emission from circumstellar envelopes ${ }^{\star}$
}

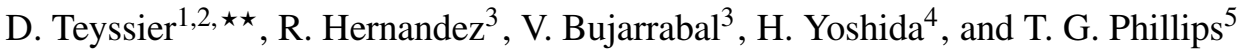

\author{
1 Departamento de Astrofísica Molecular e Infrarroja, IEM, CSIC, 28006 Madrid, Spain \\ 2 Space Research Organization Netherlands, P.O. Box 800, 9700 AV Groningen, The Netherlands \\ e-mail: teyssier@sron.rug.nl \\ 3 Observatorio Astronómico Nacional, Apartado 1143, 28800 Alcalá de Henares, Spain \\ e-mail: v.bujarrabal@oan.es \\ 4 Caltech Submillimeter Observatory, 111 Nowelo Street, Hilo, HI 96720, USA \\ e-mail: hiro@submm.caltech.edu \\ 5 California Institute of Technology, Pasadena, CA 91125, USA \\ e-mail: phillips@submm.caltech.edu
}

Received 4 July 2005 / Accepted 30 December 2005

\section{ABSTRACT}

Aims. We present the results of a multi-transition CO observational program conducted on a sample of AGB and post-AGB stars envelopes. We have collected maps and single pointing observations of these envelopes in 5 rotational transitions ranging from $J=1-0$ to $J=6-5$, including in particular new observations of the CO line at $691 \mathrm{GHz}$ at the CSO. The use of such a set of mm and submm CO line on stellar envelopes is rare and limited to the work of some authors on IRC+10216.

Methods. Using a model for the CO emission of an AGB circumstellar envelope, in combination with a standard LVG approach, we have conducted a systematic modelling analysis using the whole set of CO data collected for a sample of 12 sources. We simultaneously fit all five transitions, taking into account the spatial information provided by the maps.

Results. We find mass-loss rates in the range $1 \times 10^{-7}$ to $4 \times 10^{-4} M_{\odot} / \mathrm{yr}$, and envelope temperatures ranging from $20 \mathrm{~K}$ to $1000 \mathrm{~K}$ at a radius of $10^{16} \mathrm{~cm}$. There seem to be a general anti-correlation between mass loss rates and temperature, the high mass loss rate AGBs having low temperatures, and vice versa. We show that most AGB data can be fitted using a constant mass loss rate, at least within the calibration uncertainties associated with the data collected at different frequencies. For some cases though (e.g. CIT 6, R Hya, $\chi$ Cyg), a change in the mass loss rate history needs to be invoked to reconcile data at low- and high- $J$, a scenario already mentioned by several authors to explain observations of WX Psc.

Key words. stars: AGB and post-AGB - stars: circumstellar matter, mass-loss - infrared: stars

\section{Introduction}

Observations of mm-wave $\mathrm{CO}$ lines are probably the best tool to measure the main properties of circumstellar envelopes around AGB stars. The ${ }^{12} \mathrm{CO}$ and ${ }^{13} \mathrm{CO} J=1-0$ and $J=$ 2-1 lines, at $\mathrm{mm}$ wavelengths, have been systematically observed in AGB envelopes, often just yielding the total emitted flux, but in some cases also including high-resolution mapping (see e.g. Loup et al. 1993; Neri et al. 1998, etc.). The total extent of the CO-rich envelope is thought to be given by the photodissociation radius, $R_{\mathrm{co}}$, beyond which the interstellar UV photons significantly destroy $\mathrm{CO}$. The size of the envelope may however also be due in some cases to variations in the mass loss rate with time. Depending on the dissociating

* Figures 4-23 and Appendix are only available in electronic form at http: //www . edpsciences.org

$\star \star$ Currently at ESAC, Villafranca del Castillo, Spain. photon absorption by dust and, mainly, by the outer CO molecules themselves, $R_{\text {co }}$ ranges in actual objects between about $10^{16} \mathrm{~cm}$, in diffuse shells, and a few $10^{17} \mathrm{~cm}$, in the thickest ones (Mamon et al. 1988). These low- $J$ lines have been particularly useful for measuring mass loss rates, averaged over the time needed to form such envelopes, between $\sim 300$ and 10000 yr. The measurement procedures apparently are quite robust (see the compilation of data and discussion on the methods by e.g. Loup et al. 1993; Bujarrabal 2004).

The efficient measurement of the circumstellar mass from $\mathrm{CO}$ lines is essentially made possible by their low excitation requirements and by the simple chemistry of $\mathrm{CO}$, whose abundance is quite constant across the envelope (except for the above mentioned photodissociation effects) and from object to object (except for moderate differences between C-rich, O-rich and S-type stars). Precisely because of the easy excitation of the 
$J=1-0$ and 2-1 lines, they are not very useful for estimating circumstellar temperatures, at least in a systematic way (they can be used for such a task only when very accurate mapping is available and the lines are optically thick). Moreover, the outer layers, close to $R_{\mathrm{co}}$, largely dominate the total emission of these lines, and it is very difficult to learn about the properties of regions inside $r \sim 10^{16} \mathrm{~cm}$. Such regions are particularly important for understanding mass ejection by AGB stars: they are the place where the grains form and the acceleration of the circumstellar material, probably due to radiation pressure acting onto grains, occurs. The study of the different layer properties, not considering only average values, is also necessary for detecting possible variations in the mass loss rates. This would indeed be basic for understanding the end of the AGB phase, which is thought to be due to a strong, late increase of the mass ejection rate. Strong variations of the mass loss rate have been found in some sources from CO mapping and from scattered light or FIR imaging (e.g. Mauron \& Huggins 2000; Olofsson et al. 2000), revealing that this parameter may change by significant factors during the AGB phase, following both recurrent and secular trends.

Further studies of circumstellar envelopes around AGB stars therefore require the observation of lines with higher excitation requirements, typically in the submillimetre wavelength range. The energies above the ground state of the low $\mathrm{CO}$ levels are $E(J=1)=5.5 \mathrm{~K}$ and $E(J=2)=$ $16.6 \mathrm{~K}$. On the other hand, AGB envelopes exhibit typical temperatures of order $T_{\mathrm{k}} \sim 50-100 \mathrm{~K}$ and $T_{\mathrm{k}} \sim 10 \mathrm{~K}$ at respective radius of $r=10^{16} \mathrm{~cm}$ and $r=10^{17} \mathrm{~cm}$. So, both $J=1-0$ and $J=2-1$ lines are easily excited in most of the envelope. In order to select regions at $r \sim 10^{16} \mathrm{~cm}$ from the star, one needs to observe at least the CO $J=4-3$ transition, for which $E(J=4)=50 \mathrm{~K}$, or better $J=6-5$, with $E(J=6)=$ $110 \mathrm{~K}$. Submillimetre lines are still difficult to observe from the ground, because of atmospheric absorption and other instrumental problems (see Sect. 2). Nevertheless, there have been several attempts to remedy this, including the wideband study of the well known source IRC +10216 by Groenewegen et al. (1998), the surveys of Young et al. (1995), Knapp et al. (1998), and the recent work by Kemper et al. (2003), that includes $\mathrm{CO}$ lines up to $J=6-5$ and $J=7-6$ in 4 sources.

In this paper we present $\mathrm{CO}$ observations in a sample of 20 stars (Sect. 2), including 14 AGB stars of C-rich, O-rich and S-type chemistry; we also tried to include relatively thick and diffuse envelopes. Two planetary nebulae (PNe), two protoplanetary nebulae (PPNe), and two yellow hypergiants were also observed. We performed in particular accurately calibrated $J=6-5$ observations, including some limited mapping. Other $\mathrm{CO}$ observations in our sources from the bibliography and archives are also discussed. The data are fitted using a molecular line emission model (Sects. 3, 4), including a study of the line intensities and profile shape and of the angular intensity distributions. We present a detailed discussion on the line emission properties and the parameters that can be derived from this kind of data. As will be shown, we have been able to study regions at distances $\lesssim 10^{16} \mathrm{~cm}$ from the central star, for which the temperature and density laws can be estimated from our data.

\section{New observations}

\subsection{Observational details}

The new observations presented in this paper were carried out at the Caltech Submillimeter Observatory (CSO) in September 2001 and February 2002. The observations concentrated mostly on the $J=6-5$ transition of CO $(691.472 \mathrm{GHz})$, although some additional spectra were recorded in the $J=$ 3-2 (345.796 GHz) and $J=4-3(461.041 \mathrm{GHz})$ transitions. All $\mathrm{CO}(6-5)$ data were obtained under very good atmospheric conditions, with zenith opacities at $225 \mathrm{GHz}\left(\tau_{z}\right)$ in the range $0.05-0.08$, resulting in system temperatures of 4000-10000 K. Whenever possible, the pointing was performed on the target itself, in a spectroscopic mode consisting in observing a 5-pt cross around the expected position of the source. When this was not possible, the closest standard pointing source was considered, resulting in somewhat larger pointing uncertainties (order $3^{\prime \prime}-5^{\prime \prime}$ ), especially when compared to the beam at $691 \mathrm{GHz}\left(10.3^{\prime \prime}\right)$. All frontends were connected to an AcoustoOptic spectrometer (AOS) providing a $1 \mathrm{MHz}$ frequency resolution element on an instantaneous bandwidth of $500 \mathrm{MHz}$. Finally, the modulation on the sky was done using the wobbler switching observing mode with secondary mirror throws of $3^{\prime}$.

A total of 20 evolved stars was observed in the $J=6-5$ transitions (see Table 1). Emission was detected in 17 sources, of which all but IRC+10216 (e.g. Crosas \& Menten 1997), NGC 7027, CRL 618 and CRL 2688 (Herpin et al. 2002) are first detections at this frequency. For a subset of sources, additional spectra were obtained at offset positions from the centre, mainly to assess the eventual extent of the emission in this transition. The corresponding spectra are shown in Figs. 2 to 23.

\subsection{Calibration}

Special attention was given to the absolute calibration of the data. At the CSO, the calibration is done using the chopper wheel technique (Penzias \& Burrus 1973) in its simplest approach, i.e. assuming that the atmosphere, the hot load and the cabin temperatures are similar (the advantage of this technique being the non-dependence on the atmospheric transmission to first order). If this assumption is only marginally met, one can show that the difference between the initial antenna temperature and the corrected value writes (see Wang et al. 1994 for a complete discussion):

$\frac{T_{\mathrm{A}, \mathrm{corr}}^{*}}{T_{\mathrm{A}}^{*}}-1 \propto \frac{J_{\mathrm{hot}}-J_{\mathrm{ATM}}}{J_{\mathrm{ATM}}} \times\left[1+\exp \left(\tau_{z} A\right)\right]$

where $J_{\text {hot }}$ and $J_{\mathrm{ATM}}$ are the respective hot and atmosphere Rayleigh-Jeans temperatures at the frequency of interest, and $A$ the airmass at a given elevation. This correction becomes significant at high frequencies since opacities get higher, especially if observations cannot be performed at high elevations. The absolute correction factor was computed using a dedicated feature of the ATM atmospherical model tool developed by Pardo and Cernicharo (Cernicharo 1985; Pardo 1996). The atmospheric opacities were directly measured during the observations by mean of a dedicated receiver available at 
Table 1. Various parameters for the sources modelled in this paper. References for the distances: ${ }^{a}$ based on Hipparcos measurements, ${ }^{b}$ Bujarrabal et al. (1994), ${ }^{c}$ from Groenewegen et al. (1998), ${ }^{d}$ from Schöier \& Olofsson (2001), ${ }^{e}$ from Castro-Carrizo et al. (2001). ${ }^{f}$ González-Delgado et al. (2003). The star temperatures correspond to color-temperatures in the IR; in dubious cases, we chose a standard value of $2000 \mathrm{~K}$. Note however that this temperature has little influence on the excitation since the total intensity at 4.7 micron is directly taken from the observations. The IR fluxes at $4.7 \mu \mathrm{m}$ are inferred from Gezari et al. (1999).

\begin{tabular}{lccccc}
\hline \hline Source & $\begin{array}{c}\text { Coordinates } \\
(\text { Eq. 2000.0) }\end{array}$ & Chemis. & $\begin{array}{c}\text { Distance } \\
(\mathrm{pc})\end{array}$ & $\begin{array}{c}\text { Star } \\
\text { Temp. (K) }\end{array}$ & $\begin{array}{c}\text { IR Flux } \\
(\mathrm{Jy})\end{array}$ \\
\hline IK Tau & $03: 53: 28.611: 24: 20.0$ & $\mathrm{O}$ & $250^{f}$ & 2000 & 1800 \\
R Leo & $09: 47: 33.511: 25: 43.6$ & $\mathrm{O}$ & $100^{a}$ & 2600 & 3300 \\
IRC+10216 & $09: 47: 57.313: 16: 44.0$ & $\mathrm{C}$ & $120^{c}$ & 2000 & 11000 \\
CIT 6 & $10: 16: 02.030: 34: 19.0$ & $\mathrm{C}$ & $440^{d}$ & 1300 & 1700 \\
Y CVn & $12: 45: 07.845: 26: 24.9$ & $\mathrm{C}$ & $220^{a}$ & 1500 & 450 \\
R Hya & $13: 29: 42.8-23: 16: 52.8$ & $\mathrm{O}$ & $200^{a}$ & 2600 & 2110 \\
RX Boo & $14: 24: 11.625: 42: 13.4$ & $\mathrm{O}$ & $156^{a}$ & 2000 & 1600 \\
IRC+20370 & $18: 41: 54.817: 41: 08.0$ & $\mathrm{C}$ & $700^{b}$ & 1500 & 745 \\
IRC+10420 & $19: 26: 48.111: 21: 17.0$ & $\mathrm{O}$ & $5000^{e}$ & 6200 & 180 \\
$\chi$ Cyg & $19: 50: 33.932: 54: 33.9$ & $\mathrm{~S}$ & $150^{b}$ & 2000 & 3000 \\
CRL 3068 & $23: 19: 12.417: 11: 35.0$ & $\mathrm{C}$ & $1000^{b}$ & 2000 & 50 \\
LP And & $23: 34: 28.043: 33: 02.0$ & $\mathrm{C}$ & $630^{c}$ & 2000 & 600 \\
\hline
\end{tabular}

the CSO. On average, the corrections were in the range 5-10\% at $461 \mathrm{GHz}$ and $10-20 \%$ at $691 \mathrm{GHz}$, depending on the source elevation and opacity at the time of observation.

The second additional correction we made consisted in applying an antenna gain dependence with the elevation, again an effect most critical at the highest frequencies. At the CSO, this correction factor is not systematically calibrated and monitored, so we performed dedicated observations of IRC+10216 at various elevations, and fitted the following equation to the data (von Hörner \& Wong 1975; Greve et al. 1998):

$G(\mathrm{el})=\exp \left[-\left(\frac{4 \pi R \sigma_{\mathrm{g}}(\mathrm{el})}{\lambda}\right)^{2}\right]$

$\lambda$ being the wavelength (in $\mu \mathrm{m}$ ) and $R$ a factor (here $\sim 0.8$ ) accounting for the antenna shape, and with

$$
\begin{aligned}
\sigma_{\mathrm{g}}(\mathrm{el})^{2}= & {\left[\sigma(0)\left(\cos (\mathrm{el})-\cos \left(\mathrm{el}_{\mathrm{o}}\right)\right)\right]^{2} } \\
& +\left[\sigma(90)\left(\sin (\mathrm{el})-\sin \left(\mathrm{el}_{\mathrm{o}}\right)\right)\right]^{2}
\end{aligned}
$$

where the gain dependence is entirely determined at all wavelengths by $\mathrm{el}_{\mathrm{o}}$ (the elevation at gain unity), $\sigma(0)$ and $\sigma(90)$, the $\mathrm{rms}$ surface deviation to the ideal paraboloid at elevations el $=0$ and $\mathrm{el}=90$ degrees respectively. $\mathrm{el}_{\mathrm{o}}$ is a design parameter of the antenna, which is set to 50 degrees at the CSO (Tom Phillips, private communication), allowing to fit our limited data sample despite of the lack of data at lower elevations. The fit indicates $\sigma(0) \simeq \sigma(90) \simeq 43 \mu \mathrm{m}$, also in good agreement with theoretical predictions from the mechanical antenna model (D. Woody private communication).

Finally, the data corrected following the steps described above were converted to main-beam antenna temperatures $\left(T_{\mathrm{mb}}\right)$ using beam efficiencies derived from observations of Mars and Jupiter. We derive beam efficiencies of 0.65, 0.45 and 0.55 at 345,461 and $691 \mathrm{GHz}$ respectively, as well as Half Power Beam Width (HPBW) of 20.6", 15.5" and 10.3" respectively. Adding up pointing uncertainties, these result in effective (smeared) HPBWs of $21^{\prime \prime}, 16^{\prime \prime}$ and $12^{\prime \prime}$ respectively.
The overall calibration uncertainties considered for the CSO data set are $20 \%$ at 345 and $461 \mathrm{GHz}$, and $30 \%$ at $691 \mathrm{GHz}$.

\section{Data from other papers}

In complement to the new data obtained at the CSO, we made use of the CO $J=1-0$ and $J=2-1$ survey of AGB stars by Neri et al. (1998). The dataset provided by this study contains extended mapping of most of the targets considered in the present paper. The unpublished spectra used in the present work were kindly made available by R. Neri. In some cases, the absolute calibration was revisited to be consistent with additional spectra we have been collecting on our side at the $\left(0^{\prime \prime}, 0^{\prime \prime}\right)$ position. The beam sizes assumed for the two corresponding CO transitions are $23^{\prime \prime}$ and $13^{\prime \prime}$ respectively. In addition, some spectra of the two lower- $J$ transitions of $\mathrm{CO}$ were also taken from the survey by Gonzalez-Alfonso et al. (1998). At higher frequencies, we made extensive use of the JCMT data archive (http://cadcwww.hia.nrc.ca/jcmt/) to fetch spectra of the $J=3-2$ and $J=4-3$ transitions. Finally, most of the missing spectra at these latter frequencies could be found in the surveys by Young et al. (1995) and Knapp et al. (1998). While published data were taken at face values, the calibration of the JCMT archive data is a priori less certain. We assumed that all data were provided in the $T_{\mathrm{A}}^{*}$ scale (JCMT staff, private communication) and calibrated them to main beam temperatures using efficiencies of 0.63 and 0.52 at 345 and $461 \mathrm{GHz}$ respectively. The respective beam sizes assumed for these transitions are $15.5^{\prime \prime}$ and $12^{\prime \prime}$.

In summary, the data sample considered in this paper makes use of the first five $\mathrm{CO}$ rotational transitions available from the ground (i.e. except $J=5-4$ ) for 10 sources, while 2 are limited to the three lowest $J$ lines. This is not the total collected sample since some of the sources could not be treated with the simple model used in this study (due to e.g. multiple winds, or a later evolutionary stage) and will be addressed in a forthcoming paper. 


\section{Molecular emission model}

Our results have been fitted using a model for the $\mathrm{CO}$ emission of an AGB circumstellar envelope (see a description of a first version of the code in Bujarrabal et al. 1989). The excitation of the molecular energy levels is treated using a standard LVG approach, which is relatively well suited for our envelopes, in which the expansion velocity is significantly larger than the local velocity dispersion. The LVG code was only used to estimate the level excitation for different distances from the star. The level populations were used to calculate the absorption and emission coefficients for different radial velocities (and assuming the above described dynamics), the standard radiative transfer equation was solved with them for a number of impact parameters up to the outer radius. The brightness distribution so obtained was convolved with Gaussian telescope beams (Sects. 2 and 3), and then directly compared to the observed profiles. The local dispersion is supposed to be given by turbulence, a value of $\sim 0.2 \mathrm{~km} \mathrm{~s}^{-1}$ for this parameter is found to be compatible with the observed profile shapes. We adopted a constant expansion velocity $V$ across the envelope. This assumption is justified for the relatively outer layers we are able to probe $\left(\gtrsim 10^{15} \mathrm{~cm}\right)$, in which the radial velocity is supposed to be constant due to the strong decrease of the forces with distance from the central star (e.g. Kwok 1975; Goldreich $\&$ Scoville 1976). This fact clearly points out the main problem in the treatment of radiative transfer in AGB envelopes: the absorption of IR photons emitted by the star and the central dust condensation (that may produce vibrational excitation of molecules and subsequent rotational excitation in the vibrational ground state) cannot be treated locally, and absorption by all molecules between these inner regions and the treated layer must in principle be taken into account. This is a real problem, not just an artifact of the escape probability formalism, since its treatment requires a very good knowledge of the velocity field (to estimate at which radii the various values of the radial velocity lose radiative connexion), which is certainly not available in such detail. In the escape probability formalism, the radial coherence length is given by the logarithmic velocity gradient $\mathrm{d}(\ln (V)) / \mathrm{d}(\ln (r))=\frac{r}{V} \frac{\mathrm{d} V}{\mathrm{~d} r}$; we will assume for this parameter a value $\sim 0.1$, which yields long absorption paths in the radial direction (as is certainly the case in actual envelopes), but not larger than the radius of the treated shell, $r$, which would be in conflict with the LVG treatment itself. We have checked that the dependence of the results on the values of the logarithmic velocity gradient is not significant as long as its values are kept moderate. Values of $\frac{r}{V} \frac{\mathrm{d} V}{\mathrm{~d} r} \lesssim 0.01$ do introduce, however, a significant decrease of the excitation in the outer layers of thick and extended envelopes; fortunately, the outer radius of most sources is small enough to guarantee that the emission of the lines is mainly due to collisions and the adopted value of the logarithmic velocity gradient has little effect on the rotational line emission. The IR emission from the inner regions is directly taken from measurements at wavelengths close to that of the $\Delta v=1 \mathrm{CO}$ transition, $4.7 \mu \mathrm{m}$ (and corrected for the assumed distance to the star). $V$ is a free parameter in our modelling, usually well determined from the profile width.
The lowest 40 rotational levels in the three lowest vibrational states are considered. An approximate correction of the effects of neglecting levels in the $v=0$ state with significant population, i.e. spurious overpopulation of the treated levels and collisional under-excitation of low- $J v>0$ levels, is introduced, although it is small even in the innermost shells.

Since our main goal is the determination of the physical conditions in the probed shells, we have assumed parameterized reasonable laws for the temperature and density, even if this implies a lack of coherence in the description of the thermodynamics (see discussion by e.g. Schöier et al. 2001). We have assumed that the kinetic temperature varies with radius like $T=T_{\mathrm{o}}\left[\frac{10^{16}}{r(\mathrm{~cm})}\right]^{\alpha}+T_{\infty}$; a maximum temperature, $T_{\max } \sim$ $1000 \mathrm{~K}$, is imposed in the inner regions. We note that theoretical and empirical studies often agree with this law and yield $\alpha \sim 1$ (see Goldreich \& Scoville 1976; Justtanont et al. 1994; Groenewegen et al. 1999, etc.). As we will see our results are also compatible with this law. The number density is assumed to be $n=\frac{\dot{M}}{4 \pi r^{2} V m}$, deduced from the mass conservation law for given values of the mass loss rate, $\dot{M}$, and the expansion velocity, $V ; m$ is the mass of the typical gas particle, assumed to be $m \sim 3 \times 10^{-24} \mathrm{gr}$, since the gas is thought to be mainly molecular in AGB envelopes. $T_{\mathrm{o}}, \alpha, T_{\infty}$, and $\dot{M}$ are free parameters deduced from the fitting of observed profiles and maps.

The total extent of the $\mathrm{CO}$ envelope, $R_{\mathrm{co}}$, must be compatible with the $\mathrm{CO}$ photodissociation theory (see Mamon et al. 1998). We have taken $R_{\text {co }}$ as a free parameter, since it could be smaller than the photodissociation radius if the mass loss rate has only lasted a short time, and since it may vary depending on the interstellar UV field for each source and several circumstellar parameters (not only on the mass-loss rate, also on the expansion velocity, $\mathrm{CO}$ abundance, and grain number density and size); we recall that the calculations by Mamon et al. were basically performed for the conditions in the carbon rich star IRC+10216. We have in any case kept in mind the photodissociation radius expected from theoretical grounds. The values of the outer $\mathrm{CO}$ radius deduced from our model fitting for most of the sample stars are compatible with the Mamon et al.'s calculations within $\sim 30 \%$, in spite of the sometimes difficult adaptation of the calculations to the various cases. In two O-rich objects, IK Tau and R Hya (which shows a clear time evolution of the ejection properties) the outer radius is about $60 \%$ of our expectations from the theory. The radius of CRL 3068 is about one half of the expected one, perhaps because the star has been ejecting gas at the present high rate only in the last $\sim 7 \times 10^{3}$ years. The envelope around the yellow hypergiant IRC +10420 is very different from those of AGB stars and can be hardly compared with the theoretical calculations.

As mentioned above (Sect. 1), the chemistry of CO is relatively simple and well understood. The $\mathrm{CO}$ abundances, $X_{\mathrm{co}}$, are assumed to be constant across the envelope, up to $R_{\mathrm{co}}$, beyond which $X_{\mathrm{co}}=0 . X_{\mathrm{co}}$ is assumed to be equal to $8 \times 10^{-4}$ in C-rich envelopes (except for IRC +10216 , for which we took $X_{\mathrm{co}}=1.1 \times 10^{-3}$, Sect. 6 , since for this source we mainly tried to reproduce the results by Groenewegen et al. 1999), equal to $3 \times 10^{-4}$ for O-rich sources, and equal to $6 \times 10^{-4}$ for S-type stars. See discussions on the values of this parameter by e.g. 
Bujarrabal et al. (1989) and Loup et al. (1993). Note that in most cases the changes of the values of $X_{\mathrm{co}}$ can be almost exactly compensated by opposite changes in the mass loss rates.

In our code, it is possible to introduce different laws depending on the distance to the star, following the suggestion by e.g. Kemper et al. (2003) that the mass loss rate could strongly vary with time, and subsequently the density and temperature laws could vary with $r$. However, we have tried to keep constant the laws across the whole envelope as far as possible, since a very high number of input parameters obviously introduces redundancy in the fitting and the meaning of the estimates is not always clear. We note that in our model we have in practice four main parameters $\left(V, \dot{M}, T_{\mathrm{o}}\right.$, and $\left.\alpha\right) . T_{\max }$ and $T_{\infty}$ have less influence and $R_{\text {co }}$ and $X_{\text {co }}$ are in some way not independent of the others. The inner radius $R_{\mathrm{i}}$ is assumed to be small enough to have no influence on the results (except for some exceptional case, Sect. 6). $V$ is mostly given by the profile width, and the other parameters serve to explain the intensities and extent of the five observed lines.

Not all the lines yield really "independent" data, since sometimes two lines have similar excitation requirements and are observed with similar beamwidths; this is in particular not the case of the $J=2-1$ and $J=1-0$ lines that are observed with very different beams and do in fact not probe the same regions. Generally speaking, the low- $J$ data are very useful to estimate the total mass loss rate and their emission depends on $R_{\text {co. }}$. The $J=6-5$ line is on the other hand very sensitive to the temperature and mainly probe regions closer than $\sim 10^{16} \mathrm{~cm}$. As it is well known, the line shape essentially depends on the opacity: two-horn profiles indicate optically thin, spatially resolved emission; parabolic profiles indicate optically thick unresolved lines etc. In summary, even if the density and temperature (for $r$ ranging between about $10^{15}$ and $10^{17} \mathrm{~cm}$ ) actually follow more complex laws than what we have assumed or present very sharp and strong variations, we will just try to measure "average" values, instead of undertaking a fitting procedure with very uncertain results. As we will see, our observational data only indicated a two-component model in two sources; in both cases (particularly for R Hya) the composite nature of the profiles already suggested a composite envelope. A third case could have been considered (IK Tau), as suggested e.g. by a radial cut in the $J=2-1$ transition (Fig. 15). However, the lack of high- $J$ transitions for this source made it particularly difficult to accurately assess the parameters associated with the innermost component. Finally, in the case of $\chi \mathrm{Cyg}$, the model suggested a significant mass-loss rate drop for radii $\lesssim 5 \times 10^{15} \mathrm{~cm}$ (Sect. 6).

We have taken from the bibliography the values of other needed parameters in the fitting procedure, namely the distance, IR emission at $4.7 \mu \mathrm{m}$ and general stellar properties. See Table 1 and discussion source by source in Sect. 6 . The results of our model fitting are discussed in Sect. 6.

\section{General properties of the rotational emission of $\mathrm{CO}$}

In this section we will approximately describe the emission of the $\mathrm{CO}$ rotational transitions in AGB circumstellar envelopes by means of quite simple formulae. We will see by comparison with the numerical results that this description is usually accurate. These formulae also will help us to understand the numerical results obtained from our code.

\subsection{Analytical formulae}

The analytical equations approximately describing the rotational emission of the $\mathrm{CO}$ molecule in circumstellar envelopes (CSE) are deduced in Appendix A. The first (and most important) result from this discussion is that a very simple analytical description of the $\mathrm{CO}$ line emission, both as a function of radius and in total, can be attained.

In this formulation, we have distinguished optically thin from optically thick shells. In both cases, the total emission (quantified in number of photons emitted per second in a shell, $n_{\text {ph }}$ ) can be easily parameterized by introducing for each $J$ a radius $r_{\text {exc }}$ (Eqs. (A.7) and (A.22)). This radius separates two areas, that we call under-excited $\left(r \gg r_{\mathrm{exc}}\right)$ and overexcited $\left(r \ll r_{\text {exc }}\right)$. $r_{\text {exc }}$ basically depends on some of the model free parameters, and on energies associated to each transition. Depending on the radius probed, we show in Appendix A that the emission depends on the temperature, density and radius following simple laws (in some cases being in fact independent of some of these parameters), and scales with power laws of $J$. In both cases, the emission in the under-excited region drops sharply following an exponential law. Since $r_{\text {exc }}$ decreases with increasing $J$, we find again that only low- $J$ lines can be emitted in the outermost layers of the envelope, while only high- $J$ lines are able to efficiently probe the conditions in inner layers.

We summarize in Tables 5 and 6 the dependence on the main parameters resulting from our analysis, detailed in the Appendix. Figure 1 illustrates these results for both optically thin and optically thick cases. We recall that our formulae have been deduced using the simple laws mentioned in this paper for the physical conditions (Sect. 4), so that we have further assumed, in order to derive several formulae, that $T \underset{\sim}{\propto} 1 / r$ (i.e. $\alpha \simeq$ 1 ); we have also assumed that the contribution of the very inner shells (with constant $T$ ) to the total emission is negligible, which is not valid for FIR transitions with higher values of $J$.

In the following subsection, we compare these simple radial profiles to the emission calculated via our numerical model.

\subsection{Comparison with detailed calculations}

The values of the frequency integrated fluxes of the $\mathrm{CO}$ lines from our circumstellar emission model are very similar to those predicted by the simple formulae deduced in Sects. A.1 and A.2. As examples of optically thin emission we can take the calculations of R Leo and RX Boo, while CRL 3068 and LP And are examples of envelopes with optically thick CO lines (Sect. 6). We must remember that the optically thin or thick nature of the lines is clearly indicated by the shape of the lines (Sect. 4).

The calculations of the emission per unit shell, $n_{\mathrm{ph}}\left(\mathrm{cm}^{-1} \mathrm{~s}^{-1}\right)$, are quantitatively compatible with the predictions of our simple formulae, both in the optically thin and 

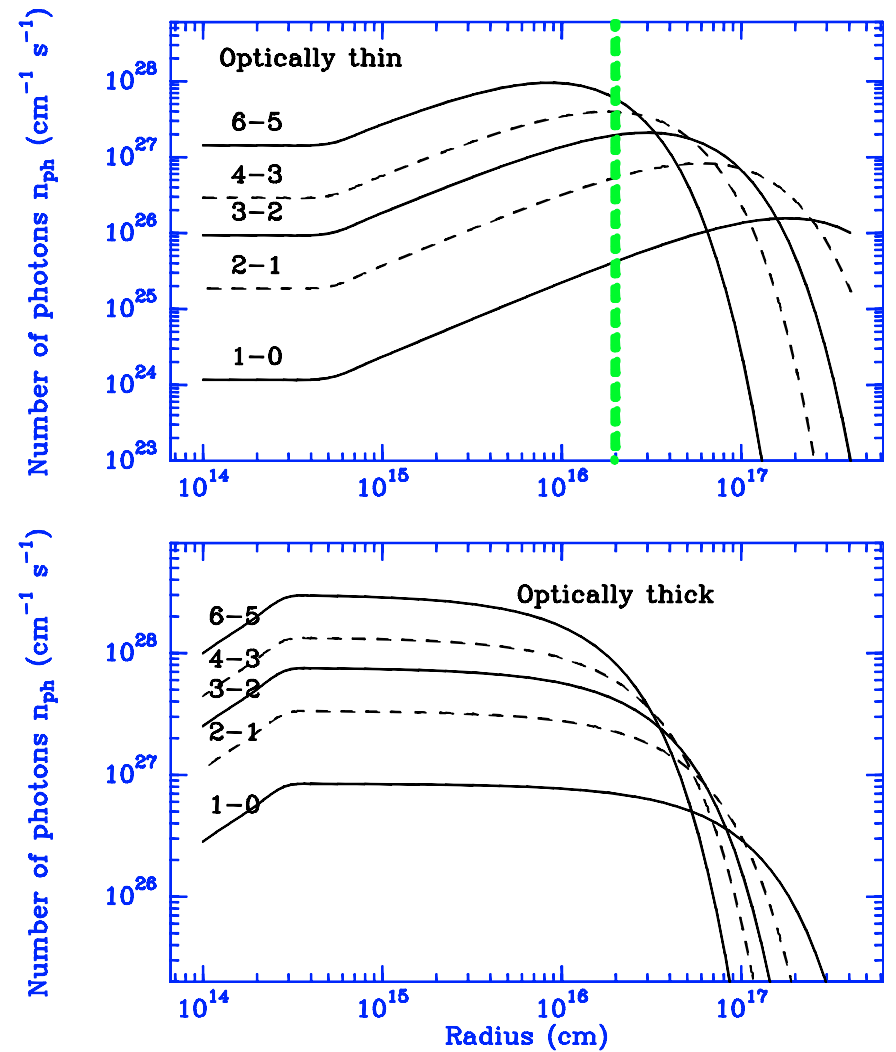

Fig. 1. Plot of the $\mathrm{CO}$ rotational emission (in number of photons per second and per unit shell) according to the simple laws computed in Appendix A. Note that the three segments have been plotted following strictly the expressions compiled in Tables 5 and 6 , so that connections between the various areas are not as smooth as in reality. The dashed-vertical line in the upper panel indicates a representative photodissociation radius for low mass-loss envelopes.

thick cases, see Eqs. (A.4), (A.15). It is easy to check that introducing the fitted parameters (Table 3 ) in the above formulation we in general reproduce the model results displayed in Figs. 4 to 14.

Only in the cases of weak emission in low-density layers, some discrepancies between the above simple formulae and the detailed calculations may appear, due to that the actual excitation temperatures are lower than the kinetic ones. We have in particular noticed (A.1) that in the optically thin case the transition radius, $r_{\mathrm{exc}}$, from over-excitation to under-excitation, occurs at shorter distances than expected from its simple definition, Eq. (A.7). Another effect appears in this case: the intensity in regions closer than $r_{\mathrm{exc}}$ is somewhat stronger than suggested by the simple formula, mainly because the actual partition function is smaller than in the thermalized case.

The total number of photons emitted by the envelope, $n_{\mathrm{ph}, \text { tot }}\left(\mathrm{s}^{-1}\right.$, given by our simple Eqs. (A.6), (A.8), (A.23), (A.24)) is also compatible with that obtained from our detailed model calculations, being therefore compatible with the observations for the physical conditions determined from our model fitting. We must remember the conversion of the photon
Table 2. Line parameters for the new observations performed at the CSO. $T_{\mathrm{mb}}$ and the associated uncertainty correspond to the respective spectrum peak intensity and noise after smoothing to a $1 \mathrm{~km} \mathrm{~s}^{-1}$ resolution. This may be not representative of the overall spectrum intensity in cases of double-horn profile shapes. $V_{\mathrm{FW}}$ stands here as the equivalent line width of a star envelope of integrated intensity $I_{\mathrm{mb}}$ and intensity $T_{\mathrm{mb}}$, and is simply computed as the ratio of these two quantities. For flat-topped spectra, this is close to the spectra full width between the first nulls. ${ }^{a}$ Cases of non detection. The upper limit is given as $1 \sigma$ here.

\begin{tabular}{l|c|cccc}
\hline \hline Source & $\begin{array}{c}\text { CO } \\
\text { line }\end{array}$ & $\begin{array}{c}T_{\mathrm{mb}} \\
(\mathrm{K})\end{array}$ & $\begin{array}{c}V_{\mathrm{LSR}} \\
\left(\mathrm{km} \mathrm{s}^{-1}\right)\end{array}$ & $\begin{array}{c}V_{\mathrm{FW}} \\
\left(\mathrm{K} \mathrm{km} \mathrm{s}^{-1}\right)\end{array}$ \\
\hline O Ceti & $J=6-5$ & $6.2 \pm 1.1$ & 47.0 & 8.2 & 51.0 \\
CRL 618 & $J=6-5$ & $2.8 \pm 0.4$ & -25.0 & 30.0 & 83.4 \\
R Leo & $J=6-5$ & $3.9 \pm 0.6$ & -0.5 & 7.9 & 31.0 \\
CIT 6 & $J=3-2$ & $4.8 \pm 0.3$ & -1.4 & 24.5 & 117.6 \\
& $J=4-3$ & $5.5 \pm 0.6$ & -2.1 & 20.7 & 113.8 \\
& $J=6-5$ & $7.8 \pm 0.7$ & -1.8 & 21.5 & 167.7 \\
V Hya & $J=6-5$ & $2.4 \pm 0.5$ & -17.0 & 24.5 & 58.7 \\
Y CVn & $J=6-5$ & $1.3 \pm 0.4$ & 20.0 & 12.2 & 15.8 \\
R Hya & $J=3-2$ & $1.5 \pm 0.2$ & -10.0 & 9.5 & 14.3 \\
& $J=6-5$ & $6.0 \pm 0.5$ & -10.0 & 8.8 & 53.0 \\
RX Boo & $J=6-5$ & $2.5 \pm 0.6$ & 0.0 & 13.9 & 34.8 \\
X Her & $J=3-2$ & $1.4 \pm 0.1$ & -73.0 & 7.4 & 10.3 \\
& $J=6-5$ & $1.7 \pm 0.3$ & -72.0 & 8.1 & 13.8 \\
IRC+20370 & $J=6-5$ & $<0.27^{a}$ & - & - & - \\
AFGL2343 & $J=6-5$ & $<0.32^{a}$ & - & - & - \\
IRC+10420 & $J=6-5$ & $1.5 \pm 0.4$ & 66.0 & 54.0 & 81.0 \\
M1-92 & $J=6-5$ & $<0.19^{a}$ & - & - & - \\
$\chi$ Cyg & $J=6-5$ & $2.0 \pm 0.3$ & 10.0 & 11.9 & 23.8 \\
V Cyg & $J=6-5$ & $1.6 \pm 0.4$ & 15.0 & 11.4 & 18.2 \\
CRL 2688 & $J=6-5$ & $11.0 \pm 1.1$ & -35.0 & 28.7 & 317.0 \\
NGC7027 & $J=6-5$ & $10 \pm 0.4$ & 25.0 & 25.0 & 250.0 \\
CRL 3068 & $J=6-5$ & $1.8 \pm 0.4$ & -31.5 & 13.7 & 24.6 \\
LP And & $J=6-5$ & $3.0 \pm 0.4$ & -17.0 & 18.0 & 54.0 \\
R Cas & $J=6-5$ & $1.9 \pm 0.3$ & 27.0 & 12.9 & 24.6 \\
\hline & & & & &
\end{tabular}

emission rate, $n_{\text {ph,tot }}\left(\mathrm{s}^{-1}\right)$, to detectable energy flux, $F\left(\mathrm{erg} \mathrm{s}^{-1} \mathrm{~cm}^{-2}\right)$ or $F\left(\right.$ watts $\left.\mathrm{m}^{-2}\right)$, for a distance $D$ :

$F=n_{\mathrm{ph}, \mathrm{tot}} \frac{h v}{4 \pi D^{2}}$.

Only for optically thin lines we must take into account the effects of the relatively low excitation mentioned above. The total emitted flux depends on $r_{\mathrm{exc}}^{2}$ in this case, so that a proper determination of this parameter from detailed modeling is useful. This effect is partially compensated by the stronger emission per volume unit due to relatively low rotational temperatures, the result being a moderate underestimate of the total emission when our simple formulae are used. Methods to deduce more accurately the values of $r_{\text {exc }}$ and the emissivity for actual diffuse envelopes will be presented in a forthcoming paper.

From our simple formulae we can conclude that the determination of the mass loss rate (i.e. the determination of $n_{\mathrm{o}}$ ), can only be properly done when the lines are not very optically thick. Fortunately, this is almost always the case for the $J=1-0$ line (see the above mentioned cases of LP And 
Table 3. Best fit parameters. ${ }^{a}$ For these sources, a model with two components is used, separated in two areas on the envelope. See text for details.

\begin{tabular}{lcccccccc}
\hline \hline Source & $R_{\mathrm{i}}$ & $R_{\mathrm{co}}$ & $\dot{M}$ & $X(\mathrm{CO})$ & \multicolumn{2}{c}{$V_{\text {exp }}$} & \multicolumn{3}{c}{ Temperature profile } \\
& $\left(10^{14} \mathrm{~cm}\right)$ & $\left(10^{14} \mathrm{~cm}\right)$ & $\left(M_{\odot} / \mathrm{yr}\right)$ & $\left(10^{-4}\right)$ & $\left(\mathrm{km} \mathrm{s}^{-1}\right)$ & $T_{\mathrm{o}}(\mathrm{K})$ & $\alpha$ & $T_{\infty}(\mathrm{K})$ \\
\hline IK Tau & 1 & 600 & $4.7 \times 10^{-6}$ & 3 & 18 & 40 & 1.2 & 3 \\
R Leo & 1 & 130 & $1.2 \times 10^{-7}$ & 3 & 6 & 100 & 1.0 & 3 \\
IRC+10216 & 1 & 3000 & $1.2 \times 10^{-5}$ & 11 & 13 & 35 & 1.2 & 3 \\
CIT 6 $^{a}$ & 1 & 100 & $5.0 \times 10^{-6}$ & 8 & 12 & 90 & 1.3 & 3 \\
& 100 & 5000 & $7.5 \times 10^{-6}$ & 8 & 16.5 & 35 & 1.2 & 3 \\
Y CVn $_{\text {R Hya }}{ }^{a}$ & 1 & 400 & $1.0 \times 10^{-7}$ & 8 & 6.5 & 90 & 0.9 & 3 \\
& 1 & 50 & $0.4 \times 10^{-7}$ & 3 & 1.5 & 300 & 0.8 & 3 \\
RX Boo $_{\text {IRC+20370 }}$ & 1 & 180 & $3.0 \times 10^{-7}$ & 3 & 6.5 & 400 & 0.8 & 3 \\
IRC+10420 & 100 & 420 & $2.2 \times 10^{-7}$ & 3 & 7.5 & 90 & 1.0 & 3 \\
$\chi$ Cyg & 20 & 5500 & $7.0 \times 10^{-6}$ & 8 & 13.5 & 20 & 0.9 & 6 \\
CRL 3068 & 1 & 5000 & $3.0 \times 10^{-4}$ & 3 & 37.0 & 1000 & 1.2 & 3 \\
LP And & 1 & 3000 & $6.0 \times 10^{-5}$ & 8 & 13.5 & 45 & 0.9 & 3 \\
\hline
\end{tabular}

and CRL 3068). At this respect, the theoretical relation between $\dot{M}$ and $R_{\text {co }}$ and observational data from the (optically thin) ${ }^{13} \mathrm{CO}$ emission must often be used.

The temperature and its variation with the radius can be well determined from the intensity of the different lines, since $r_{\text {exc }}$ varies with $J$ (in the optically thin case, we must first calculate the mass-loss rate). Data on high- and low- $J$ transitions allow then an empiric study of $T_{\mathrm{k}}(r)$, in particular high- $J$ lines are necessary to probe the innermost layers. The determination of the temperature from line intensity ratios may be difficult in both the optically thin are thick regimes, since the line ratios may not depend on the temperature law (cf. Appendix). We would then need the comparison of a high- $J$ transition (at least the $J=4-3$ line) and the $J=1-0$ or 2-1 lines, provided that the former is optically thick and $J=1-0$ is optically thin or that $R_{\text {co }}<r_{\text {exc }}$ for low values of $J$. For example, a favorable situation takes place when all transitions are optically thick and $R_{\text {co }}<r_{\text {exc }}(1-0)$, then the intensity ratio $J-J-1 / 1-0$ (for $J \geq 4$ ) is just proportional to $J^{3} T_{\mathrm{o}}$.

A future paper will be devoted to a deeper discussion on the analysis of the excitation of submm and FIR molecular lines in AGB circumstellar envelopes and to the determination of the circumstellar parameters from such data using simple analytical expressions.

\section{Individual sources}

We discuss here the fitting results for each of the sources considered in this study. Apart from the four main free parameters introduced in Sect. 4, an additional degree of freedom was taken into account, resulting from the absolute calibration uncertainties associated with each line. We have thus allowed the modelled emission to be corrected for each line by a given coefficient varying within the ranges of this uncertainty. The error bars considered here are respectively 15\%, 15\%, 20\%, 20\% and $30 \%$ for the five transitions (in increasing numbers of $J$ ). Table 4 gathers the coefficients applied to the best fit spectra for
Table 4. Calibration coefficients used to fit the data displayed in Figs. 2 to 23. The mean of these coefficients is also given for each transition, with its standard deviation. Note how this later increases with the frequency, which is expected as calibration uncertainties get worse at higher- $J$.

\begin{tabular}{lccccc}
\hline \hline Source & \multicolumn{5}{c}{ CO transition } \\
& $J=1-0$ & $J=2-1$ & $J=3-2$ & $J=4-3$ & $J=6-5$ \\
\hline IK Tau & 1.05 & 0.9 & 1.1 & - & - \\
R Leo & 1.0 & 0.87 & 1.1 & 1.0 & 1.0 \\
IRC+10216 & 0.9 & 0.95 & 1.1 & 0.9 & 1.1 \\
CIT 6 & 0.85 & 0.85 & 1.05 & 0.9 & 1.15 \\
Y CVn & 1.0 & 1.0 & 1.05 & 1.15 & 1.0 \\
R Hya & 1.1 & 0.85 & 1.15 & - & 0.8 \\
RX Boo & 1.1 & 1.05 & 1. & 0.85 & 1.15 \\
IRC+20370 & 1.05 & 0.87 & 1.1 & 1.0 & - \\
IRC+10420 & 0.9 & 1.0 & - & 1.1 & 1.2 \\
X Cyg & 0.94 & 0.89 & 1.05 & 1.05 & 0.7 \\
CRL 3068 & 1.12 & 1.02 & 1.0 & 1.1 & 0.8 \\
LP And & 1.0 & 1.12 & 0.84 & 0.95 & 0.95 \\
\hline Mean & $\mathbf{1 . 0 0}$ & $\mathbf{0 . 9 5}$ & $\mathbf{1 . 0 5}$ & $\mathbf{1 . 0 0}$ & $\mathbf{1 . 0 2}$ \\
(rms) & $\mathbf{( 0 . 0 9 )}$ & $\mathbf{( 0 . 0 9 )}$ & $\mathbf{( 0 . 0 8 )}$ & $\mathbf{( 0 . 9 )}$ & $\mathbf{( 0 . 1 4 )}$ \\
\hline & & & & &
\end{tabular}

each source and transition. On average, the correction remain very reasonable (and close to 1), especially in comparison with other unknowns associated to the model (cf. Sect. 4). As expected, larger scatters are observed at higher frequencies. It is interesting to note some systematic trends, like e.g. the fact that in most cases, the modelled $J=2-1$ emission is too intense, while the $J=3-2$ one is too weak. It could indicate a systematic over- or under-estimate of the efficiencies used at the corresponding telescopes, but could also point towards a more fundamental caveat in the model itself. In the following sections we discuss in more details the results of the model fitting for each source. 
Table 5. Dependence of the number of photons emitted by unit shell $\left(n_{\mathrm{ph}}\right.$, in $\left.\mathrm{cm}^{-1} \mathrm{~s}^{-1}\right)$, on the physical conditions and distance to the star, from the analytical description of the CO line emission given in Appendix A, for the optically thin and optically thick cases. The $r \ll r_{\text {exc }}$ (overexcited) area is separated in two zones, the innermost layers corresponding to the cases where $T_{\text {rot }}$ is constant and equal to $T_{\max }$ (cf. Sect. 4). $E_{j}$ is here the energy of the upper level of the transition, $J$, and $\Delta E$ stands for $E_{j}-E_{j-1}$.

\begin{tabular}{|c|c|c|c|}
\hline \multirow{2}{*}{$\begin{array}{l}\text { Radiative } \\
\text { Case }\end{array}$} & \multicolumn{2}{|c|}{ Over-excited } & Under-excited \\
\hline & $\begin{array}{c}\text { Innermost } \\
\text { layers }\end{array}$ & $\begin{array}{c}\text { Intermediate } \\
\text { layers }\end{array}$ & $\begin{array}{c}\text { Outermost } \\
\text { layers }\end{array}$ \\
\hline $\begin{array}{l}\text { Opt. thin } \\
\text { Opt. thick }\end{array}$ & $\begin{array}{c}n_{\mathrm{ph}} \propto J^{4} n_{\mathrm{o}} X_{\mathrm{o}} / T_{\mathrm{o}} \\
n_{\mathrm{ph}} \propto J^{2} r T_{\mathrm{o}} V\end{array}$ & $\begin{array}{c}\propto J^{4} r n_{\mathrm{o}} X_{\mathrm{o}} / T_{\mathrm{o}} \\
\propto J^{2} T_{\mathrm{o}} V\end{array}$ & $\begin{array}{c}\propto \frac{J^{4} r n_{0} X_{\mathrm{o}}}{T_{\mathrm{o}}} \mathrm{e}^{-E_{j} / T_{\text {rot }}} \\
\propto J^{3} r T_{\mathrm{o}} V \mathrm{e}^{-\Delta E / T_{\text {rot }}}\end{array}$ \\
\hline
\end{tabular}

Table 6. Total number of photons emitted by CO lines in a circumstellar envelope, $n_{\mathrm{ph}, \mathrm{tot}}\left(\mathrm{s}^{-1}\right)$, from the simple analytical description given in Appendix A, for the optically thin and optically thick cases. The cases is which the CO photodissocation radius, $R_{\mathrm{co}}$, is smaller or greater than $r_{\text {exc }}$ must be treated separately. (Remember that $r_{\text {exc }}$ is defined to be equal to $\frac{T_{\mathrm{o}} r_{\mathrm{o}}}{E_{j}}$ in the optically thin case and equal to $\frac{T_{\mathrm{o}} r_{\mathrm{o}}}{\Delta E}$ in the opaque case; again, $E_{j}$ is here the energy of the upper level of the transition and $\Delta E=E_{j}-E_{j-1}$.)

\begin{tabular}{l|cc}
\hline \hline Radiative & $R_{\mathrm{co}} \gg r_{\text {exc }}$ & $R_{\text {co }}<r_{\text {exc }}$ \\
case & Extended CO shell & Small CO shell \\
\hline Opt. thin & $n_{\text {ph,tot }} \propto \frac{J^{4} n_{0} X_{0}}{T_{\mathrm{o}}} r_{\mathrm{exc}}^{2} \stackrel{\propto}{\sim} \frac{J^{4} n_{0} X_{0} T_{0}}{[J(J+1)]^{2}}$ & $\propto \frac{J^{4} n_{0} X_{0}}{T_{\mathrm{o}}} R_{\mathrm{co}}^{2}$ \\
Opt. thick & $n_{\mathrm{ph}, \mathrm{tot}} \propto J^{3} V r_{\mathrm{exc}}^{2} \propto J V T_{\mathrm{o}}^{2}$ & $\propto J^{3} V r_{\mathrm{exc}} R_{\mathrm{co}} \propto J^{2} V T_{\mathrm{o}} R_{\mathrm{co}}$ \\
\hline
\end{tabular}

\subsection{C-rich stars}

\subsubsection{IRC+10216}

In order to gauge our model against existing tools, we run simulations of the well-studied envelope of IRC+10216. Our goal was not to refine the best-fit parameters for this source but rather to check whether plugging in reported parameters would result in a consistent model. We used the parameters reported by Groenewegen et al. (1998), derived from a more sophisticated model than ours; we note for instance that Groenewegen et al. introduced a fully non-local treatment of radiative transfer and calculations of the temperature from modelling of the circumstellar thermodynamics. The comparison between both models was for this reason not obvious. In particular, it was somewhat difficult to figure out how these authors deal with the IR flux of the central star and dust grains. We decided to rely on the flux at $4.7 \mu \mathrm{m}$ compiled by Gezari et al. (1999), translating into a flux of $11000 \mathrm{Jy}$. The treatment of transfer of the stellar emission across their model envelope is also difficult because of the very low velocity gradient, we tried to simulate this effect in our calculations using a logarithmic velocity gradient as low as $\sim 0.02$. For the rest, we used the parameters corresponding to the $L=10000 L_{\odot}$ model of Groenewegen et al. (1998), and shown in their Fig. 3. We assumed a distance of $120 \mathrm{pc}$. Our inputs are gathered in Table 3 and the best fit is illustrated in Fig. 2.

The comparison of both models, between them and with the observations, is satisfactory. Our synthetic profiles are within $10 \%$ of those reported by Groenewegen et al. (1998), and allow a good fit of the data in all probed transitions, again within a $10 \%$ factor. We confirm that the model slightly overestimates the $J=1-0$ line, while it under-estimates the $J=$ 3-2 one (see Table 4 for the calibration coefficients used to adjust the model to the spectra). Our $J=6-5$ data cannot be directly compared to that of these authors since we used a different telescope (the CSO). Despite of the theoretically smaller beam of the JCMT, our line is stronger than that used by Groenewegen et al. (1998), essentially due to significant error beam loss at the JCMT at this frequency. Note that our spectrum is consistent with that reported by Crosas \& Menten (1997). We also show in Fig. 3 how our model reproduces spectra obtained at offset positions in the two highest transitions used here.

\subsubsection{LP And}

LP And (IRC+40540) has been observed and studied in various CO transitions by Schöier \& Olofsson (2001). As can be seen in their Fig. 4, the spectra used by these authors are very similar to the ones considered in the present study (see Fig. 14). Using submm lines up to $J=4-3$, they report a best fit mass-loss rate of $1.5 \times 10^{-5} M_{\odot} / \mathrm{yr}$ with an external radius of $4 \times 10^{17} \mathrm{~cm}$ at a distance of $630 \mathrm{pc}$. In the formalism used in this paper, their optimal temperature law translates into $\alpha=0.9$ and $T_{\mathrm{o}}=30 \mathrm{~K}$. We show in Figs. 14 and 23 the result of our model when plugging in their best fit parameters. After some minor parameter changes, we find that all 5 lines up to $J=6-5$ can be reasonably fitted assuming $\dot{M}=1.2 \times 10^{-5} M_{\odot} / \mathrm{yr}$, and $R_{\mathrm{CO}}=5 \times 10^{17} \mathrm{~cm}$. The calibration coefficients are within $10 \%$ of unity, with the exception of the $J=3-2$ transition which is over-estimated by almost $20 \%$. When compared to the theoretical plots of Fig. 1, the modelled photon emission is consistent with an optically thick envelope, also evidenced by the parabolic shape of most profiles.

The model also allows a fit of the radial cuts derived from the maps of Neri et al. (1998) in the $J=1-0$ and $J=2-1$ transitions. The model is also consistent with the $\mathrm{CO}(6-5)$ spectrum obtained at an offset position of $5^{\prime \prime}$ (Fig. 23), showing essentially that the material emitting in this transition is pointlike. We have compared our model against spectra taken with other telescopes, and published by Schöier \& Olofsson (2002). 

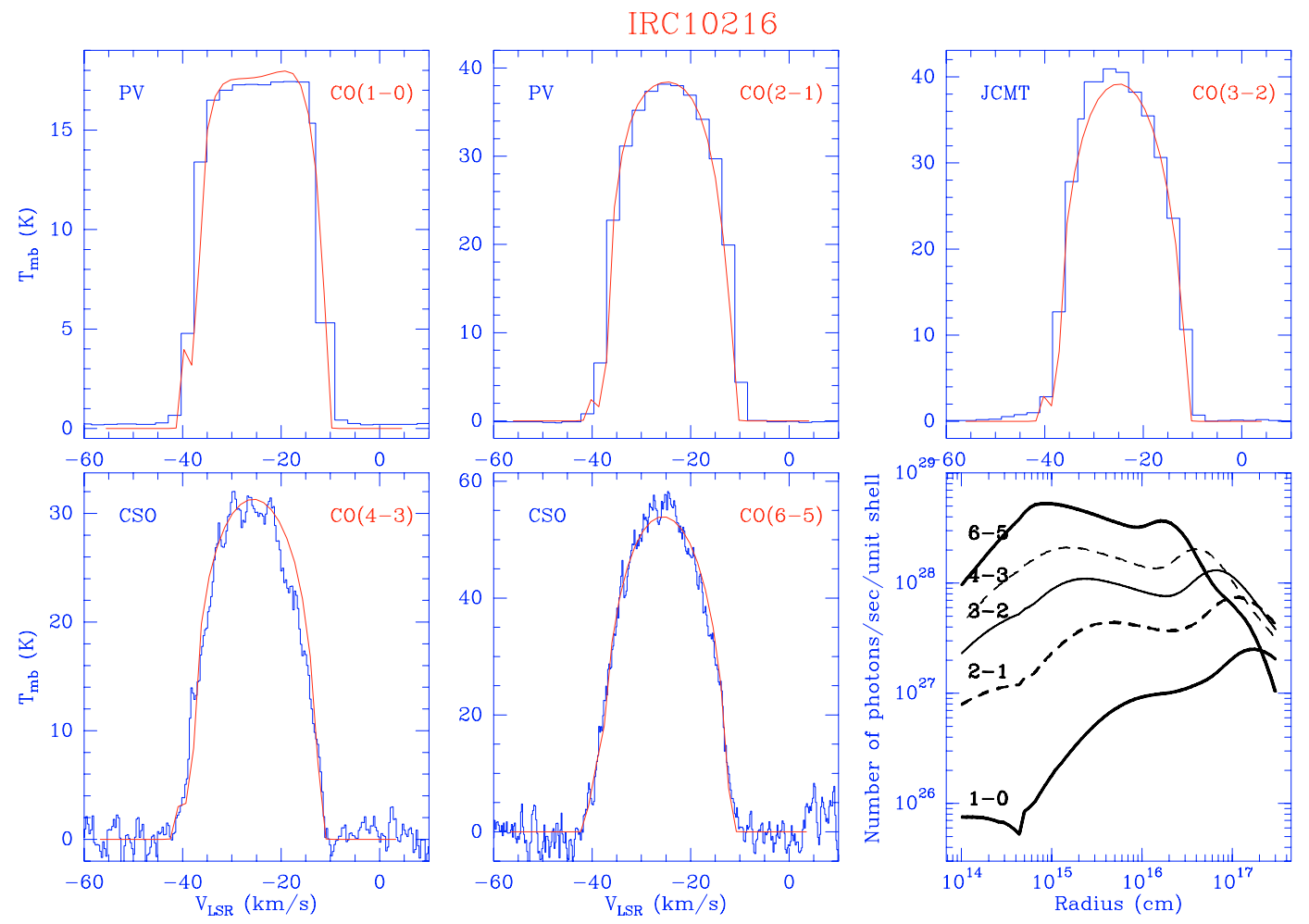

Fig. 2. Best model fit to the available set of data at offset $\left(0^{\prime \prime}, 0^{\prime \prime}\right)$ for IRC+10216, using input parameters published by Groenewegen et al. (1996, see text). For each of the panels, we show the CO transition and the telescope used to obtain the spectrum displayed. All temperatures are in $T_{\mathrm{mb}}$. The lower right panel plots plots as a function of the distance to the star the number of photons emitted by a given transition per second and per unit shell layer. It illustrates the contribution of the different layers to the emission.
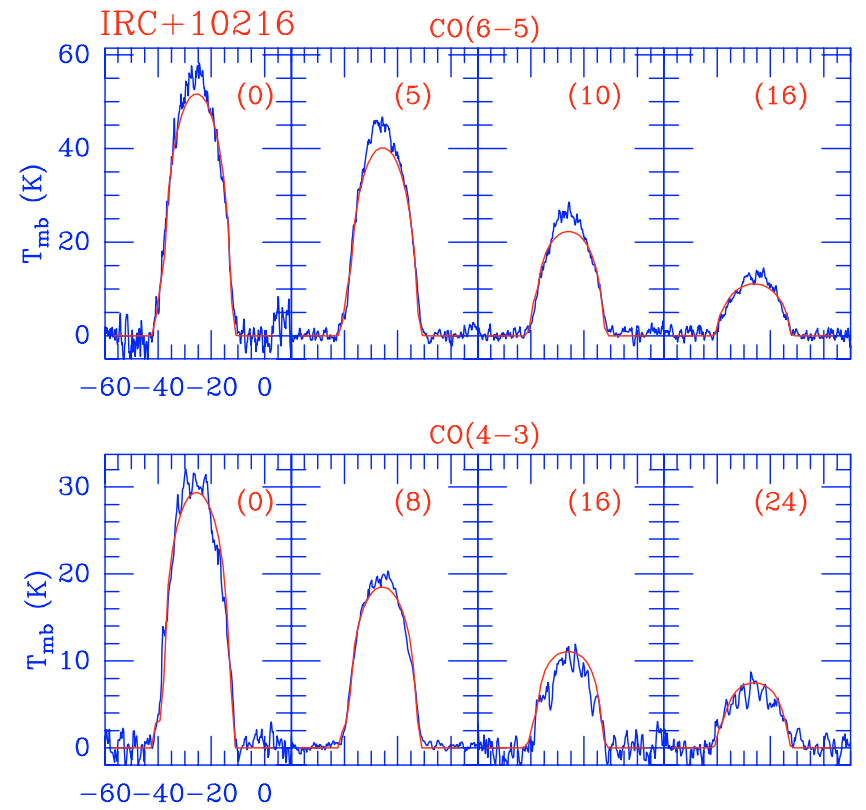

Fig. 3. Best fit model to offset positions of IRC +10216 taken in the $\mathrm{CO}(4-3)$ and $\mathrm{CO}(6-5)$ transitions with the CSO. The numbers between parenthesis in the upper right corner of each box indicates the spatial offset from the $(0,0)$ position.

We found very good agreement with all various beams, showing the good quality of the modelled source size and the likelihood of the spherical symmetry assumption.
The mass loss rate derived in our model is very similar to that obtained by Woods et al. (2003). However, Knapp et al. (1998) and Loup et al. (1993) deduced a slightly smaller value of $\dot{M}$, the difference is of about a factor of 2 . Probably the reason of the discrepancy is the low temperature deduced from our fitting, expected for the high mass-loss rate of this source.

We have obtained a particularly complete and accurate set of data in LP And. The fitting of these spectra is very good, in spite of the very simple model used: we can state that, at least in this source, the $\mathrm{CO}$ emission from $J=1-0$ to $J=6-5$ can be very properly described by a source model with a very limited number of parameters, in particular a constant mass-loss rate during the last $10^{4} \mathrm{yr}$.

\subsubsection{CRL 3068}

The case of CRL 3068 appears to be very comparable to the one of LP And, with an optically thick envelope (Fig. 13), a relatively high mass-loss rate of $\dot{M}=6 \times 10^{-5} M_{\odot} / \mathrm{yr}$, and a similar temperature law. Assuming a distance of $1000 \mathrm{pc}$, the external radius is found to be $R_{\mathrm{CO}}=5 \times 10^{17} \mathrm{~cm}$. This parameter allows to fit well the extended emission mapped in the first two CO transitions, and shown in Fig. 22. To first order, the $J=6-5$ transition is over-estimated by $25 \%$ by our model. However, the spectra has low $\mathrm{S} / \mathrm{N}$ ratio $(\sim 4)$ and the calibration coefficient was chosen to best fit the line profile shape on average. 
Our mass-loss rate is three times larger than that found by Woods et al. (2003), from detailed fitting of data of ${ }^{12} \mathrm{CO}, J=$ $1-0$ to $J=4-3$, and ${ }^{13} \mathrm{CO}, J=1-0$ to $J=3-2$. We included in our analysis the $J=6-5$ transition and maps of the $J=1-0$ and 2-1 transitions. Almost a factor 2 can be explained by the larger distance and $\mathrm{CO}$ abundance assumed by those authors. The rest of the difference is certainly due to the low temperature deduced by us in the outer envelope. Such a low temperature is imposed in our calculations to explain the low emission from $J=2-1$ found in the outer envelope, where most of the circumstellar mass is placed. CRL 3068 is a prototype of very cold high-mass envelopes (e.g. Omont et al. 1993). Our value of the mass-loss rate is very similar to that found by Loup et al. (1993) and smaller by a factor of 2 than that by Sopka et al. (1989). We think that the (moderate) differences found for CRL 3068 illustrate the uncertainties (or better, the accuracy) of the determination of the mass-loss rates in AGB envelopes.

\subsubsection{Y CVn}

Compared to LP And and CRL 3068, Y CVn has relatively weak emission, with lines of quite similar intensities in all transitions (when scaled to a common beam size). The $J=1-0$ line is however weaker by about a factor 2 , implying a relatively small external radius $R_{\mathrm{CO}}=4 \times 10^{16} \mathrm{~cm}$ at a distance of $220 \mathrm{pc}$. The low mass-loss rate found $\left(\dot{M}=1 \times 10^{-7} M_{\odot} / \mathrm{yr}\right)$ is also consistent with an optically thin envelope, as is confirmed by the profiles of the low- $J$ transitions, as well as the overall photon emission distribution over the radius (Fig. 7). The model requires absolute temperatures 2-3 times larger than the high mass-loss stars mentioned above (Table 3 ). This is mainly triggered by the $J=6-5$ line, found to be in excess of $1 \mathrm{~K}$. For this latter transition, the line shape is only marginally fitted, a discrepancy partly explained by the poor $\mathrm{S} / \mathrm{N}$ ratio $(\sim 3)$ of the measurement. Again, the best model is found to fit the mapping data shown in Fig. 17 reasonably well. Note the relatively high temperature found in this source, expected for its relatively low mass-loss rate.

Again our mass-loss estimates are very similar to those derived by Schöier et al. $(2001,2002)$ and by Knapp et al. (1998); all these authors used the same distance assumed here. Older estimates by Loup et al. (1993) and Wannier \& Sahai (1986) are somewhat higher than ours, but the difference is mostly due to the larger distance assumed by those authors (the derived mass-loss rates roughly scales with the distance).

\subsubsection{IRC+20370}

IRC+20370 (CRL 2232) appears as an intermediate case in our C-rich star sample, with a mass-loss rate of $\dot{M}=7 \times$ $10^{-6} M_{\odot} /$ yr. As seen in Fig. 10, the emission is marginally thin in the $J=1-0$ transition, with a flat-topped line profile, but it gradually becomes optically thick as $J$ increases and one penetrates into the envelope. For the higher $J$ this is only visible from the modelled photon emission as we have no data above the $J=3-2$ transition. The maps of Neri et al. (1998) reveal a somewhat extended envelope in low- $J$ transitions (Fig. 19), implying a relatively large external radius $R_{\mathrm{CO}}=3.5 \times 10^{17} \mathrm{~cm}$ at $D=700 \mathrm{pc}$. Interestingly, the temperature slope drops again with $\alpha=0.9$, but a quite lower envelope temperature is needed (Table 3). Also in this source, all lines are so easily thermalized that an increase of $\dot{M}$ has almost no effect on the transitions probed. The only way to get all 3 transitions with sufficiently high intensities was to keep the external temperature to $6 \mathrm{~K}$ (instead of $3 \mathrm{~K}$ as in all other sources). This resulted in a $25 \%$ intensity increase.

Our mass-loss rate estimate is slightly larger than that derived by Loup et al. (1993, after correcting for the distance difference), but smaller by a factor 3 than the value by Sopka et al. (1989), who only used $J=1-0$ data. Probably the reason for this discrepancy is the low temperature deduced by us for this source, constrained by the non-detection of the $J=6-5$ line, observed down to a rms of $0.3 \mathrm{~K}$.

\subsubsection{CIT 6}

In this source we deduce that a change in the mass-loss rate and expansion velocity occurred about $250 \mathrm{yr}$ ago. Such a change, which is not dramatic in any case (Table 3 ), was necessary to explain the well-measured decrease of the intensity with distance to the centre (Fig. 16) and the composite profiles (Fig. 6). This is most striking in the $J=2-1$ radial cut where a constant mass-loss rate would result in too little emission at offsets above $\sim 15^{\prime \prime}$. Schöier et al. $(2001,2002)$ derive a mass-loss rate that is intermediate between our two values, using the same distance as taken by us. The temperature profile derived by Schöier et al. (2002) is also roughly intermediate between our curves for both regimes, although the approach to the temperature estimates by those authors (which includes calculations of the cooling and heating rates for only one component) are quite different from ours. Older estimates of the mass ejection rates by Loup et al. (1993) are also compatible with our results. The total $\mathrm{CO}$ radius is large, in order to explain the quite extended $\mathrm{CO}$ emission (detectable at least up to $45^{\prime \prime}$ equivalent to $3 \times$ $10^{17} \mathrm{~cm}$ ), but still compatible with theoretical calculations for these mass-loss rates by Mamon et al. (1988).

\subsection{O-rich stars}

\subsubsection{R Leo and RX Boo}

These two sources are described together as they share similarities in their envelope emission. As seen in Figs. 5 and 9, they both exhibit optically thin emission, but also show a red emission excess that we could only partly reproduce. It is particularly striking for R Leo, and suggests that ad hoc models would be required to account for this feature. Also, the 2-1/1-0 line ratio is relatively high (5 and 3 respectively), which implies both low mass-loss rates $\left(1.2 \times 10^{-7} M_{\odot} / \mathrm{yr}\right.$ and $2 \times 10^{-7} M_{\odot} / \mathrm{yr}$ respectively) and a small external radius $\left(R_{\mathrm{CO}}=1.3 \times 10^{16} \mathrm{~cm}\right.$ at $100 \mathrm{pc}$, and $R_{\mathrm{CO}}=4.2 \times 10^{16} \mathrm{~cm}$ at $156 \mathrm{pc}$, respectively).

For both sources, the $J=6-5$ line is observed to be relatively bright, implying higher temperatures than in most $\mathrm{C}$-rich stars reported above. For R Leo, $T_{\mathrm{o}}=100 \mathrm{~K}$ and $\alpha=1.0$ offers a good compromise to keep the $J=1-0$ weak enough to meet 
the high $2-1 / 1-0$ ratio (combined with the small $R_{\mathrm{CO}}$ ), while boosting the line emission from the innermost layers. On the other hand, it is still difficult to find a good temperature law for RX Boo: while $\alpha$ cannot be increased without decreasing the $J=1-0$ transition, $T_{\mathrm{o}}=90 \mathrm{~K}$ is only a compromise allowing both $J=4-3$ (over-estimated by the model) and $J=$ 6-5 (under-estimated by the model) calibration adjustment coefficients to have reasonable values (respectively 0.85 and 1.2 , Table 4).

Modelling results for R Leo have been published by several authors. Scaled to our assumed distance, Bujarrabal et al. (1989) report $\dot{M}=2.5 \times 10^{-7} M_{\odot} / \mathrm{yr}$ and $R_{\mathrm{CO}}=0.8 \times 10^{16} \mathrm{~cm}$. Similarly, Knapp et al. (1998) found $\dot{M}=9.4 \times 10^{-8} M_{\odot} / \mathrm{yr}$, while the non-LTE model of González Delgado et al. (2003) yields $\dot{M}=1.5 \times 10^{-7} M_{\odot} / \mathrm{yr}$ and $R_{\mathrm{CO}}=1.3 \times 10^{16} \mathrm{~cm}$. All these estimates are very consistent with our findings.

This good agreement holds for RX Boo, at least compared to the numbers reported by Knapp et al. $\left(\dot{M}=3 \times 10^{-7} M_{\odot} / \mathrm{yr}\right)$. This is less true for the best-fit model published by Olofsson et al. (2002), $\dot{M}=7 \times 10^{-7} M_{\odot} / \mathrm{yr}$ and $R_{\mathrm{CO}}=3.7 \times 10^{16} \mathrm{~cm}$. Apart from a possible difference in the temperature law used by these authors, this factor of 3 discrepancy could arise for several reasons. First of all, we assumed an expansion velocity of $7.5 \mathrm{~km} \mathrm{~s}^{-1}$, while Olofsson et al. used $V_{\exp }=9.3 \mathrm{~km} \mathrm{~s}^{-1}$. Thus for a given spectrum peak intensity, and all other parameters being fixed, a model with larger expansion velocity requires a higher mass loss rate. As is seen in Fig. 9, using a larger $V_{\text {exp }}$ is not justified by the line-width of the observational dataset. Also, we used a higher value of the relative $\mathrm{CO}$ abundance, $3 \times$ $10^{-4}$ instead of $2 \times 10^{-4}$, which could explain the remaining difference with these authors' results.

\subsubsection{IRC+10420}

IRC +10420 is the prototype of massive and luminous yellow hypergiant stars, and it is believed to experience a transitional phase from the red to blue stages (Jones et al. 1993; de Jager 1998). As such, it may be one of the few supernova progenitors studied to date. Based on molecular data, Castro-Carrizo et al. (2001) confirmed the high mass loss rate of order $4 \times$ $10^{-4} M_{\odot} / \mathrm{yr}$ (at a distance of $5 \mathrm{kpc}$ ) already reported by several authors (e.g. Loup et al. 1993; Kastner \& Weintraub 1995; Neri et al. 1998). The shell is probably detached, with an inner ratio of $\sim 1.25 \times 10^{16} \mathrm{~cm}$, as deduced from its FIR emission and high-resolution NIR observations of the dust environment of the star (Ridgway et al. 1986; Hrivnak et al. 1989).

Figure 11 displays the spectra used in our modelling and the best-fit results. All spectra exhibit a blue excess that we did not try to fit with our model. Following the above results, we truncated the inner radius to $1 \times 10^{16} \mathrm{~cm}$ and used a massloss rate of $3 \times 10^{-4} M_{\odot} / y$. The model offers a reasonable fit to all lines but the $J=3-2$ transition, whose predicted intensity lies at least a factor of 2 below the observed intensity. We however suspect the calibration of this observation (taken from the JCMT archive) to be in error, as suggested by the dataset published by Oudmaijer et al. (1996) who report an average (excluding the blue excess) peak temperature of $2.5 \mathrm{~K}$.
A very high temperature of $T_{\mathrm{o}}=1000 \mathrm{~K}$ was required in order to be consistent with the intense $J=6-5$ observations at various offsets (Fig. 20). Using temperatures $\lesssim 100 \mathrm{~K}$ at $10^{16} \mathrm{~cm}$, as usually found in AGB envelopes, would result in high- $J$ line intensities too low by a factor of at least 5 . The deduced temperature law is accurately consistent with the high temperature found by Castro-Carrizo et al. at a distance $\sim 10^{17} \mathrm{~cm}(\sim 55 \mathrm{~K})$, necessary to explain the high brightness observed in $\mathrm{SiO}$ line emission. One major discrepancy should be pointed out: our model uses an external radius of $R_{\mathrm{CO}}=4.5 \times$ $10^{17} \mathrm{~cm}$, much smaller than that reported by Castro-Carrizo et al. That large outer radius was deduced just assuming that the $\mathrm{CO}$ shell is limited by photodissociation, but it is obvious that the shell radius could instead be given by the onset of the high mass-loss process and then be significantly smaller. Our smaller outer radius value is compatible with the total shell radius measured in recent Plateau de Bure observations (Quintana-Lacaci et al., in preparation).

\subsubsection{IK Tau}

IK Tau could be the O-rich counterpart of the phenomenon observed in CIT 6. The overall line profiles are strongly suggestive of optically thin emission, which is confirmed by the modelled photon distribution over the envelope (Fig. 4). However, a close look at the $J=2-1$ and $J=3-2$ spectra reveals an additional parabolic component of lower expansion velocity. From the best-fit profiles, it is obvious that the model misses a part of the emission located in this component. This is also striking when looking at the extended emission cuts (Fig. 15): as for CIT 6, the single-component model drops too fast and falls below the $J=2-1$ radial emission profile. Since no data were available for higher- $J$ transitions, we did not consider models with more than one component as we would have no way to check whether this change in mass-loss history would have consistent consequences on the material yet closer to the star.

The single-component model yields $\dot{M}=4.7 \times 10^{-6} M_{\odot} / \mathrm{yr}$, and $R_{\mathrm{CO}}=6 \times 10^{16} \mathrm{~cm}$ at $D=250 \mathrm{pc}$. The temperature law is steeper than for most $\mathrm{C}$-rich and O-rich stars reported here, with $\alpha=1.2$, but comparable to what is found for the two-component model of CIT 6 . These values are in reasonable agreement with those reported by Loup et al. $(1993, \dot{M}=4.1 \times$ $\left.10^{-6} M_{\odot} / \mathrm{yr}\right)$, Bujarrabal et al. $\left(1989, \dot{M}=5.1 \times 10^{-6} M_{\odot} / \mathrm{yr}\right)$, and Sopka et al. (1989). However our best-fit mass loss rate is lower by a factor of 5 than that of Gonzalez Delgado et al. (2003), who report $\dot{M}=3 \times 10^{-5} M_{\odot} / \mathrm{yr}$ and $R_{\mathrm{CO}}=2 \times 10^{17} \mathrm{~cm}$. This time, we cannot invoke difference in expansion velocity $\left(V_{\exp }=18 \mathrm{~km} \mathrm{~s}^{-1}\right.$, versus $18.5 \mathrm{~km} \mathrm{~s}^{-1}$ in our model). We also note that the work of these authors was not devoted to CO studies and it remains unclear what line intensities they used in their modelling. Finally, it is difficult to understand that such a high mass-loss rate may lead to the clear two-horn profiles (characteristic of optically thin emission) observed in this source. Perhaps the discrepancy between both models is a result of the possibly composite nature of this envelope. 


\subsubsection{R Hya}

In this source, the high- $J$ lines show a narrow intense component that strongly suggests that the inner layers are significantly different, particularly in expansion velocity, from the rest of the envelope. We have fitted the data assuming a central component expanding at only $1.5 \mathrm{~km} \mathrm{~s}^{-1}$, which dominates the emission at high frequencies (Table 3 ). However, we note that the velocity centroid observed for both components is clearly not the same. In the model, we have just assumed different systemic velocities for the two shells. This approach is perhaps not fully satisfactory, since such a difference in the center-of-gravity velocity is unexpected; the observed asymmetry could also be due to asymmetries in the spatial distribution. Figure 8 shows the spectra obtained from this modelling with our best-fit results. The results are also compatible with offset positions observed in the $J=3-2$ and $J=6-5$ transitions (Fig. 18).

In the comparison with other authors, we mainly use our outer envelope parameters, that apply in fact to most of the source. The slightly larger value of $\dot{M}$ found by González Delgado et al. (2003) is explained by the lower CO abundance used by these authors. Knapp et al. (1998) also obtained a slightly larger mass-loss rate, $4.7 \times 10^{-4} M_{\odot} \mathrm{yr}^{-1}$, still within a factor of two around ours. However, Loup et al. derived (after correcting for the distance) a slightly lower $\dot{M} \sim$ $2.3 \times 10^{-4}$. All these values can be considered as compatible with the properties of our dominant (outer) component.

\subsection{S stars}

\subsection{1. $\chi$ Cyg}

$\chi$ Cyg is the only S-type star of our sample. The case of this source is quite peculiar among our sample since we found no convincing way to reasonably fit the $\operatorname{CO}(J=6-5)$ line with a constant mass-loss rate. Figure 12 shows the best-fit results for this source. All lines but the highest- $J$ transition are well reproduced, including the well-defined double-horn profile of the $\operatorname{CO}(J=1-0)$, showing that the envelope is optically thin and resolved in low-frequency transitions. This, and the good agreement with the radial cuts displayed in Fig. 21 give good confidence in the chosen external radius $R_{\mathrm{CO}}=6 \times 10^{16} \mathrm{~cm}$ at $D=150$ pc. Our mass-loss rate of $\dot{M}=4 \times 10^{-7} M_{\odot} /$ yr is also consistent with findings of Bujarrabal et al. (1989) and Knapp et al. (1998).

The best-fit model however over-estimates the $J=6-5$ transition by a factor of almost 3 , in spite of using a low temperature $\left(T_{\mathrm{o}}=20 \mathrm{~K}\right)$ and a low mass-loss rate. This could be due to a wrong photometric calibration of the data, but could also indicate a decrease of the mass-loss rate in the last $\sim 100 \mathrm{yr}$. Mid-IR interferometry data published by Danchi et al. (1994) and Tevousjan et al. (2004) actually suggest that the dust density could be significantly smaller in a central area of extend $\gtrsim 0.3^{\prime \prime}\left(7 \times 10^{14} \mathrm{~cm}\right.$ at $\left.150 \mathrm{pc}\right)$. In particular, Tevousjan et al. deduced that the material at a distance of $1.8 \times 10^{14} \mathrm{~cm}$ corresponds to a mass-loss rate of $4.2 \times 10^{-8} M_{\odot} / \mathrm{yr}$ (at $110 \mathrm{pc}$ ), 10 times smaller than ours. We find that assuming a mass-loss rate $\dot{M} \lesssim 1 \times 10^{-7} M_{\odot} /$ yr in the inner $5 \times 10^{15} \mathrm{~cm}$, our model reproduces the reported $\mathrm{CO}(J=6-5)$ data.

\section{Discussion and conclusions}

We have carried out observations in a sample of AGB and post-AGB stars of CO submillimetre lines, namely $J=3-2$, $J=4-3$ and $J=6-5$. We have also collected data of CO $J=$ $1-0$ to $J=4-3$ in our source sample.

The observations were modelled using an LVG approach for the line excitation across each circumstellar envelope; the resulting level populations were used to calculate expected brightness temperatures in units comparable to those of observations. The synthetic and observed profiles were compared in the direction of the central star and at the spatial offsets at which emission has been detected. The model parameters were so fitted to determine some basic properties of the observed objects, mainly the mass-loss rate, expansion velocity, $\mathrm{CO}$ photodissociation radius and temperature profile across the envelope. We have also discussed how the uncertainties in the calibration, particularly noticeable in the submillimetre wave range, affect the fitting. See details in Sects. 4 and 5. Our approach consisted in simultaneously fitting line profiles of the various transitions considered, as well as their spatial variation whenever data were available. We found that respecting the multi-dimensional picture of each source helped to keep the adjustable parameters within reasonably limited ranges.

In total, 20 objects were observed by us, see the main CO line parameters in Table 1. Twelve of these sources have been modelled, all them AGB stars except for the yellow hypergiant IRC +10420 . These objects are listed in Table 1, together with the observed coordinates and some stellar properties. We can see the parameter values determined from our model fitting in Table 3.

In most cases, the observations were reproduced using just a single component, in which only the temperature, among the above parameters, shows a significant variation with radius. The line profiles and spatial brightness distributions of two of our objects, CIT 6 and R Hya, required the introduction of two components. In CIT 6, the mass-loss rate and expansion velocity changed by moderate factors (less than $50 \%$ ) about 200 yr ago. In R Hya, a much more important change took place about $100 \mathrm{yr}$ ago; we note that modelling this source is particularly uncertain because of its very peculiar profiles. Finally, for $\chi$ Cyg, we found that a decrease of the mass-loss rate by at least a factor 4 in the last 100 years would be required to reproduce the $\mathrm{CO}(J=6-5)$ data.

We have discussed that the mass-loss rates deduced from our fitting (Sect. 6) are satisfactorily compatible within a factor two with determinations by other authors, in spite of the very different approaches and emission models used in the different papers. The reason is that the value of the mass-loss rate is mainly given by the $J=1-0$ intensity, whose calibration is quite reliable and whose intensity shows a low dependence on the kinetic temperature.

We have also determined the main parameters of the temperature variation across the envelope (Table 3 ). We assumed a potential law (Sect. 4), following theoretical and observational 
results of this parameter (e.g. Goldreich \& Scoville 1976; Groenewegen 1994). We can confirm that the usually assumed values of the kinetic temperature in circumstellar envelopes are compatible with our observations. We note in particular a general trend of the sources with high mass-loss rates to show relatively low temperatures. This result is expected from theoretical grounds (e.g. Groenewegen 1994), due to the relatively high cooling rates present in dense gas, and from empirical indications (e.g. Omont et al. 1993). Only in the yellow hypergiant IRC +10420 , whose thermodynamics may be well different to that of AGB envelopes, both high mass-loss rate and temperature are present.

As we have mentioned, the temperature laws found by us are compatible with general properties derived from theoretical studies of the circumstellar thermodynamics; but the comparison of the values found for the studied sources with other authors' results is difficult, because of the different ways to deduce or implement the temperature in the models. We also recall that the detection of changes with time of the mass-loss rate and expansion velocity is sometimes unclear. In summary, our feeling is that a significant uncertainty on these measurements persists, particularly for the inner circumstellar layers. The first reason is that the interpretation of the molecular line observations in terms of kinetic temperature (and also of mass loss variations) seems still immature. Also, these studies are hampered by the low frequencies reachable from the ground (up to CO $J=7-6$, excitation energy $\sim 150 \mathrm{~K}$, only able to select regions outer than about $10^{16} \mathrm{~cm}$ ), and with serious technical problems particularly affecting the intensity calibration. The ISO satellite provided observations on high- $J$ CO lines, from $J=14-13(\sim 570 \mathrm{~K})$. But the low sensitivity (very noisy data, even for relatively intense sources) and the absence of profile shapes (only the total intensity was in fact measured, due to the low spectral resolution) avoided systematic, reliable studies from these data. The HIFI spectrometer, on board the Herschel satellite, will provide in the near future $\mathrm{CO}$ line observations with enough spectral resolution (better than $\sim 10^{6}$ ) and sensitivity ( $\mathrm{CO}$ lines detected in a few minutes of telescope time). Accurate profiles of CO lines between $J=6-5$ and $J=16-15$ (at $750 \mathrm{~K}$ ) will be measured in wide samples of stars. We hope that these observations will stimulate thoughtful studies of the temperature profiles and general structure of AGB circumstellar envelopes.

Acknowledgements. It is a pleasure to thank the CSO staff at Hilo and Caltech for all their support during the observing runs. The authors would also like to thank R. Neri for providing us with an electronic version of his catalog of evolved stars. We finally thank the anonymous referee for constructive comments and requests. DT acknowledges partial support by the Spanish Ministerio de Ciencia y Tecnologia under grant ESP2002-01693. The Caltech Submillimeter Observatory is funded by the NSF through contract AST 99-80846. This work also made extensive use of the CDS archive.

\section{References}

Bujarrabal, V. 2004, Asymmetrical Planetary Nebulae III: Winds, Structure and the Thunderbird, ASP Conf. Ser., 313, 335

Bujarrabal, V., Gomez-Gonzales, J., \& Planesas, P. 1989, A\&A, 219, 256
Castro-Carrizo, A., Lucas, R., Bujarrabal, V., Colomer, F., et al. 2001, A\&A, 368, L34

Cernicharo, J. 1985, ATM: A Program to compute Theoretical Atmospheric Opacity for Frequencies below $1 \mathrm{THz}$, IRAM technical report

Crosas, M., \& Menten, K. M. 1997, ApJ, 483, 913

Danchi, W. C., Bester, M., Degiacomi, C. G., et al. 1994, AJ, 107, 1469

de Jager, C. 1998, A\&AR, 8, 145

Gezari, D. Y., Pitts, P. S., \& Schmitz, M. 1999, Catalog of Infrared Observations, Edition 5 (Gezari+ 1999), VizieR On-line Data Catalog: II $/ 225$

Goldreich, P., \& Scoville, N. 1976, ApJ, 205, 384

González-Alfonso, E., Cernicharo, J., Alcolea, J., \& Orlandi, M. A. 1998, A\&A, 334, 1016

Greve, A., Neri, R., \& Sievers, A. 1998, A\&AS, 132, 413

Groenewegen, M. A. T. 1994, A\&A, 290, 531

Groenewegen, M. A. T., van der Veen, W. E. C. J., \& Matthews, H. E. 1998, A\&A, 338, 491

Groenewegen, M. A. T., Baas, F., Blommaert, J. A. D. L., et al. 1999, A\&AS, 140, 197

Herpin, F., Goicoechea, J. R., Pardo, J. R., \& Cernicharo, J. 2002, ApJ, 577, 961

Hrivnak, B. J., Kwok, S., \& Volk, K. M. 1989, ApJ, 346, 265

Jones, T. J., Humphreys, R. M., Gehrz, R. D., et al. 1993, ApJ, 411, 323

Justtanont, K., Skinner, C. J., \& Tielens, A. G. G. M. 1994, ApJ, 435, 852

Kastner, J. H., \& Weintraub, D. A. 1995, ApJ, 452, L141

Kemper, F., Stark, R., Justtanont, K., et al. 2003, A\&A, 407, 609

Knapp, G. R., Young, K., Lee, E., \& Jorissen, A. 1998, ApJS, 117, 209

Kwok, S. 1975, ApJ, 198, 583

Loup, C., Forveille, T., Omont, A., \& Paul, J. F. 1993, A\&AS, 99, 291

Mamon, G. A., Glassgold, A. E., \& Huggins, P. J. 1988, ApJ, 328, 797

Mauron, N., \& Huggins, P. J. 2000, A\&A, 359, 707

Neri, R., Kahane, C., Lucas, R., Bujarrabal, V., \& Loup, C. 1998, A\&AS, 130, 1

Olofsson, H., Bergman, P., Lucas, R., et al. 2000, A\&A, 353, 583

Olofsson, H., González Delgado, D., Kerschbaum, F., \& Schöier, F. L. 2002, A\&A, 391, 1053

Omont, A., Loup, C., Forveille, T., et al. 1993, A\&A, 267, 515

Oudmaijer, R. D., Groenewegen, M. A. T., Matthews, H. E., et al. 1996, MNRAS, 280, 1062

Pardo, J. R. 1996, Ph.D. Thesis, Univ. Pierre et Marie Curie-Univ. Complutense de Madrid

Penzias, A. A., \& Burrus, C. A. 1973, ARA\&A, 11, 51

Ridgway, S. T., Joyce, R. R., Connors, D., et al. 1986, ApJ, 302, 662

Schöier, F. L., \& Olofsson, H. 2001, A\&A, 368, 969

Schöier, F. L., Ryde, N., \& Olofsson, H. 2002, A\&A, 391, 577

Sopka, R. J., Olofsson, H., Johansson, L. E. B., et al. 1989, A\&A, 210 , 78

Tevousjan, S., Abdeli, K.-S., Weiner, J., et al. 2004, AJ, 611, 466

von Hörner, S., \& Wong, W.-Y. 1975, Trans. IEEE Ant. Propagat. AP-23, 689

Wang, Y., Jaffe, D. T., Graf, U. U., \& Evans, N. L. 1994, ApJS, 95, 503

Wannier, P. G., \& Sahai, R. 1986, ApJ, 311, 335

Woods, P. M., Schöier, F. L., Nyman, L.-Å., \& Olofsson, H. 2003, A\&A, 402, 617

Young, K. 1995, ApJ, 445, 872 
D. Teyssier et al.: CO line emission from circumstellar envelopes, Online Material p 1

\section{Online Material}




\section{Appendix A: Simple formulae of the rotational emission of $\mathrm{CO}$}

We detail here the analytical expressions summarized in Sect. 5. We will first assume that the excitation of the rotational lines can be described by a single rotational temperature $T_{\text {rot }}$, i.e. we will assume that the excitation temperatures for the transitions between the significantly populated levels, $T_{\text {exc }}(J, J-1)$, are equal to a constant $T_{\text {rot }}$. Due to the low Einstein coefficients of the CO rotational transitions, $T_{\text {rot }}$ is similar to the kinetic temperature $T_{\mathrm{k}}$ (i.e. the relevant lines are more or less thermalized) for densities $n>10^{5} \mathrm{~cm}^{-3} / \tau$, where $\tau$ is the opacity of the line. We will assume in most of the discussions in this Appendix that $T_{\text {rot }} \sim T_{\mathrm{k}}$; the effects of deviations from thermalization will be briefly discussed. This approach (as others assumptions we will do, see below) is mostly valid for relatively dense envelopes and for the submm lines more than for the low- $J$ transitions, since those intermediate excitation lines are formed in inner envelope regions (with relatively high densities) and present higher opacities.

\section{A.1. Optically thin emission}

In the optically thin limit, the number of photons emitted by a CO transition $J \rightarrow J-1$ per unit volume is approximately given by:

$n_{\mathrm{ph}, \mathrm{vol}}\left(\mathrm{cm}^{-3} \mathrm{~s}^{-1}\right)=A_{j, j-1} n_{j}=A_{j, j-1} g_{j} x_{j}$.

Where $A_{j, j-1}$ is the Einstein coefficient of the transition, $g_{j}$ is the statistical weight of the upper level, $n_{j}\left(\mathrm{~cm}^{-3}\right)$ is its population, and $x_{j}$ is its population per magnetic sublevel: $x_{j}=n_{j} / g_{j}$. For this formula to be valid, it is just required that $T_{\text {rot }}$ is much larger than the brightness temperature of the background continuum at the line frequency, which is almost always the case in AGB envelopes, particularly for high- $J$ transitions.

If $T_{\text {rot }}$ is large compared to the rotational constant of $\mathrm{CO}$, $B_{\text {rot }}=2.7 \mathrm{~K}$, the partition function is equal to $B_{\text {rot }} / T_{\text {rot }}$, and

$x_{j}=\mathrm{e}^{-E_{j} / T_{\text {rot }}} B_{\text {rot }} / T_{\text {rot }} n_{\text {co }}$.

Where $E_{j}$ is the energy of the $J$-level in temperature units and $n_{\mathrm{co}}\left(\mathrm{cm}^{-3}\right)$ is the density of $\mathrm{CO}$ molecules, which depends on the total density $n$ and the $\mathrm{CO}$ fractional abundance, $n_{\mathrm{co}}=n X_{\mathrm{co}}$. From Eqs. (A.1) and (A.2), we can write the emission per unit shell at a distance from the star $r(\mathrm{~cm})$ :

$$
\begin{aligned}
n_{\mathrm{ph}}\left(\mathrm{cm}^{-1} \mathrm{~s}^{-1}\right) & =4 \pi r^{2} n_{\mathrm{ph}, \mathrm{vol}} \\
& =4 \pi A_{j, j-1} g_{j} \mathrm{e}^{-E_{j} / T_{\mathrm{rot}}} B / T_{\mathrm{rot}} n X_{\mathrm{co}} .
\end{aligned}
$$

The values of $n_{\mathrm{ph}}\left(\mathrm{cm}^{-1} \mathrm{~s}^{-1}\right)$ calculated from our model fitting are plotted in the lower right panels in Figs. 4 to 14, for the studied sources, to be compared with the approximate laws deduced here.

We are also assuming that $X_{\text {co }}$ is constant up to the photodissociation radius $\left(R_{\mathrm{co}}\right)$, that the total density is $n=n_{\mathrm{o}}\left(\frac{r_{\mathrm{o}}}{r}\right)^{2}$, and that $T_{\text {rot }}=T_{\mathrm{k}}=T_{\mathrm{o}}\left(\frac{r_{\mathrm{o}}}{r}\right)^{\alpha}$, in general with $r_{\mathrm{o}}=10^{16} \mathrm{~cm}$ and $\alpha \sim 1$ (Sect. 4). So,

$n_{\mathrm{ph}}\left(\mathrm{cm}^{-1} \mathrm{~s}^{-1}\right)=4 \pi r^{2} A_{j, j-1} g_{j} \mathrm{e}^{-E_{j} / T_{\text {rot }}} B_{\text {rot }} \frac{r^{\alpha}}{T_{\mathrm{o}} r_{\mathrm{o}}^{\alpha}} n_{\mathrm{o}} \frac{r_{\mathrm{o}}^{2}}{r^{2}} X_{\mathrm{co}}$
Equation (A.4) can be integrated up to $R_{\mathrm{co}}$. Let us assume that $\alpha=1$ in most of the envelope, then:

$$
\begin{aligned}
n_{\mathrm{ph}, \mathrm{tot}}\left(s^{-1}\right)= & \int_{0}^{R_{\mathrm{co}}} n_{\mathrm{ph}}\left(\mathrm{cm}^{-1} \mathrm{~s}^{-1}\right) \mathrm{d} r \\
= & \frac{4 \pi A_{j, j-1} g_{j} B_{\mathrm{rot}} r_{\mathrm{o}}^{2} n_{\mathrm{o}} X_{\mathrm{co}}}{E_{j}} \\
& \times\left[\frac{T_{\mathrm{o}} r_{\mathrm{o}}}{E_{j}}\left(1-\mathrm{e}^{-E_{j} R_{\mathrm{co}} / T_{\mathrm{o}} r_{\mathrm{o}}}\right)-R_{\mathrm{co}} \mathrm{e}^{-E_{j} R_{\mathrm{co}} / T_{\mathrm{o}} r_{\mathrm{o}}}\right] .
\end{aligned}
$$

If $R_{\mathrm{co}}$ is much larger than the region where the emission is significant (which is true in actual cases for submm lines, but not always for the CO $J=1-0$ and 2-1 lines),

$$
\begin{aligned}
n_{\mathrm{ph}, \mathrm{tot}}\left(s^{-1}\right) & =\frac{4 \pi A_{j, j-1} g_{j} B_{\mathrm{rot}} r_{\mathrm{o}} n_{\mathrm{o}} X_{\mathrm{co}}}{T_{\mathrm{o}}}\left(\frac{T_{\mathrm{o}} r_{\mathrm{o}}}{E_{j}}\right)^{2} \\
& =\frac{4 \pi A_{j, j-1} g_{j} B_{\mathrm{rot}} r_{\mathrm{o}} n_{\mathrm{o}} X_{\mathrm{co}}}{T_{\mathrm{o}}} r_{\mathrm{exc}}^{2} .
\end{aligned}
$$

With the definition of $r_{\text {exc }}$ :

$E_{j}=\frac{T_{\mathrm{o}} r_{\mathrm{o}}}{r_{\mathrm{exc}}}$.

In the other extreme case, $R_{\mathrm{co}}<r_{\mathrm{exc}}$,

$n_{\mathrm{ph}, \mathrm{tot}}\left(s^{-1}\right)=\frac{4 \pi A_{j, j-1} g_{j} B_{\mathrm{rot}} r_{\mathrm{o}} n_{\mathrm{o}} X_{\mathrm{co}}}{T_{\mathrm{o}}} \frac{R_{\mathrm{co}}^{2}}{2}$.

Let us consider two limit cases in more detail. In the first case, the lines are "over-excited", i.e. $E_{j} \ll T_{\text {rot }}\left(\right.$ or $\left.r \ll r_{\text {exc }}\right)$. Then the emission of a shell is

$n_{\mathrm{ph}}\left(\mathrm{cm}^{-1} \mathrm{~s}^{-1}\right)=\frac{4 \pi A_{j, j-1} g_{j} B_{\mathrm{rot}} r_{\mathrm{o}} n_{\mathrm{o}} X_{\mathrm{co}} r}{T_{\mathrm{o}}}$.

Since $A_{j, j-1} g_{j}$ varies proportionally to $J^{4}$, the dependence of $n_{\mathrm{ph}}$ with the transition and the radius becomes (in the overexcited case) just

$n_{\mathrm{ph}}\left(\mathrm{cm}^{-1} \mathrm{~s}^{-1}\right) \propto J^{4} r$

in most of the envelope, where we have assumed $\alpha \sim 1$. In the very inner regions, we have assumed $T_{\mathrm{k}}=T_{\max }$ (constant), so $n_{\mathrm{ph}}$ becomes independent of $r$ :

$n_{\mathrm{ph}}\left(\mathrm{cm}^{-1} \mathrm{~s}^{-1}\right) \propto J^{4}$.

The other limit case, of "under-excitation", $E_{j}>T_{\text {rot }}$, occurs in the outer, cold envelope. In this area, Eq. (A.4) predicts a fast decrease with radius of the emissivity, dominated by the exponential factor:

$n_{\mathrm{ph}}\left(\mathrm{cm}^{-1} \mathrm{~s}^{-1}\right) \propto J^{4} r \mathrm{e}^{-E_{j} / T_{\text {rot }}}$.

We must note that our lines are optically thin in shells with relatively low density, for which $T_{\text {rot }}$ is probably not equal to $T_{\mathrm{k}}$. It is then probable that the condition $E_{j}>T_{\text {rot }}$ ("underexcitation") may take place in regions in which $T_{\mathrm{k}}$ is still higher than $E_{j}$. Therefore, the definition of $r_{\text {exc }}$ may not be as straightforward as Eq. (A.7), and its actual value may be smaller than that given by this expression (for the same reason, the dependence of the actual $r_{\text {exc }}$ on $J$ from the code results is slightly 
weaker than that given by our simple formulae, $\left.r_{\mathrm{exc}} \propto \frac{1}{J(J+1)}\right)$. In regions closer than $r_{\text {exc }}$, the departure of the excitation temperatures $T_{\text {exc }}$ from $T_{\mathrm{k}}$ may also affect the number of emitted photons. In general, low- $J$ transitions may have values of $T_{\text {exc }}$ higher than $T_{\mathrm{k}}$ (or even negative, but always with low absolute values of the optical depth), due to the fast radiative deexcitation from higher energy levels and that the kinetic temperatures are usually much higher than the low- $J$ level energy. But the partition function is dominated by the population of intermediate excitation levels, which is described by equivalent rotational temperatures that are lower than $T_{\mathrm{k}}$. Both deviations from $T_{\mathrm{k}}$ lead to somewhat higher values of $n_{\mathrm{ph}}$ than expected from our simple formulation (the effect of the partition function being dominant, as expected), typically by a factor $\sim 2$. In summary, we have two main effects on the total line emission of relatively low excitation, present in low-density envelopes, with respect to the predictions of our simple formulae: that due to relatively low values of $r_{\mathrm{exc}}$ and that due to relatively low values of the partition function. The first effect dominates, but is partially compensated by the second one, resulting in a slightly lower total emission than expected from the above formulae. See concluding remarks in Sect. 5.2.

\section{A.2. optically thick emission}

The general form of Eq. (4) under the escape probability formalism is

$n_{\mathrm{ph}, \mathrm{vol}}\left(\mathrm{cm}^{-3} \mathrm{~s}^{-1}\right)=A_{j, j-1} g_{j} x_{j} \beta$,

where $\beta$ is the escape probability. When the opacity $\tau$ is much higher than 1 ,

$n_{\mathrm{ph}, \mathrm{vol}}\left(\mathrm{cm}^{-3} \mathrm{~s}^{-1}\right)=A_{j, j-1} g_{j} x_{j} / \tau$,

where $\tau$ is an angle-average opacity:

$\tau=\tau_{\mathrm{o}} A_{j, j-1} g_{j}\left(x_{j-1}-x_{j}\right) r / V$,

with $\tau_{\mathrm{o}}=\frac{c^{3}}{8 \pi v^{3}} 3 / 2, V$ is the (almost constant) expansion velocity and $v$ is the frequency of the transition. The factor $3 / 2$ is geometrical and holds for $\mathrm{d}(\ln (V)) / \mathrm{d}(\ln (r)) \ll 1$ (Sect. 4); this factor is in the general case (optically thick line, excepted masers) equal to $1 /[1-[1-\mathrm{d} \ln (V) / \mathrm{d} \ln (r)] / 3]$.

Equation (A.14) then yields:

$n_{\text {ph,vol }}\left(\mathrm{cm}^{-3} \mathrm{~s}^{-1}\right)=\frac{1}{\mathrm{e}^{\Delta E / T_{\text {rot }}}-1} \frac{V}{\tau_{\mathrm{o}} r}$,

and

$n_{\mathrm{ph}}\left(\mathrm{cm}^{-1} \mathrm{~s}^{-1}\right)=\frac{1}{\mathrm{e}^{\Delta E / T_{\text {rot }}}-1} \frac{4 \pi r V}{\tau_{\mathrm{o}}}$

with $\Delta E=E_{j}-E_{j-1}$.

In the over-excitation case, now defined by $\Delta E \ll T_{\text {rot }}$,

$n_{\mathrm{ph}, \mathrm{vol}}\left(\mathrm{cm}^{-3} \mathrm{~s}^{-1}\right)=\frac{T_{\mathrm{rot}}}{\Delta E} \frac{V}{\tau_{\mathrm{o}} r}=\frac{T_{\mathrm{o}} r_{\mathrm{o}}^{\alpha} V}{\Delta E \tau_{\mathrm{o}} r^{\alpha+1}}$.
Therefore, in most of the envelope, where $\alpha \sim 1$, the photon emission rate per unit shell under the regime of overexcitation is:

$n_{\mathrm{ph}}\left(\mathrm{cm}^{-1} \mathrm{~s}^{-1}\right)=\frac{4 \pi T_{\mathrm{o}} r_{\mathrm{o}} V}{\Delta E \tau_{\mathrm{o}}}$

which implies that $n_{\mathrm{ph}}\left(\mathrm{cm}^{-1} \mathrm{~s}^{-1}\right)$ is practically constant with radius and varies like $J^{2}$ from line to line:

$n_{\mathrm{ph}}\left(\mathrm{cm}^{-1} \mathrm{~s}^{-1}\right) \propto J^{2}$,

In the very inner regions, $T_{\text {rot }}$ is almost constant $\left(T_{\text {rot }} \sim T_{\max }\right.$, see Sect. 3), and

$n_{\mathrm{ph}}\left(\mathrm{cm}^{-1} \mathrm{~s}^{-1}\right)=\frac{4 \pi T_{\max } r V}{\Delta E \tau_{\mathrm{o}}}$.

In these inner regions, the photon emission rates varies proportionally with $r$ and $J^{2}$.

As for the optically thin case, in the under-excitation regime $\left(\Delta E>T_{\text {rot }}\right)$ the emissivity rapidly decreases like a negative exponential,

$n_{\mathrm{ph}, \mathrm{vol}}\left(\mathrm{cm}^{-3} \mathrm{~s}^{-1}\right) \propto \mathrm{e}^{-\Delta E / T_{\mathrm{rot}}} \frac{V}{\tau_{\mathrm{o}} r}$.

We must note that in these relatively dense envelopes the underexcitation just due to the decrease of density with radius (independently of $T_{\mathrm{k}}$, as for thin envelopes, see Sect. 5.2) is not expected. On the other hand, in the under-excitation regime, we can expect a strong decrease of the opacity in more realistic calculations, as soon as the levels are not populated enough, and the decrease of the emissivity can be still sharper than predicted in Eq. (A.21).

We can also integrate Eq. (A.16) to get the total emission of the envelope in two extreme cases, and assuming $\alpha=1$. Let $r_{\mathrm{exc}}$ now be defined by

$\Delta E=\frac{T_{\mathrm{o}} r_{\mathrm{o}}}{r_{\mathrm{exc}}}$

Then, if $R_{\mathrm{co}} \gg r_{\mathrm{exc}}$ :

$$
\begin{aligned}
n_{\mathrm{ph}, \mathrm{tot}}\left(\mathrm{s}^{-1}\right) & =\int_{0}^{\infty} n_{\mathrm{ph}}\left(\mathrm{cm}^{-1} \mathrm{~s}^{-1}\right) \mathrm{d} r=\frac{4 \pi V}{\tau_{\mathrm{o}}} \frac{\pi^{2}}{6}\left(\frac{T_{\mathrm{o}} r_{\mathrm{o}}}{\Delta E}\right)^{2} \\
& =\frac{4 \pi V}{\tau_{\mathrm{o}}} \frac{\pi^{2}}{6} r_{\mathrm{exc}}^{2} .
\end{aligned}
$$

If $R_{\mathrm{co}} \ll r_{\text {exc }}$ :

$n_{\mathrm{ph}, \mathrm{tot}}\left(\mathrm{s}^{-1}\right)=\frac{4 \pi T_{\mathrm{o}} r_{\mathrm{o}} V}{\Delta E \tau_{\mathrm{o}}} R_{\mathrm{co}}=\frac{4 \pi V}{\tau_{\mathrm{o}}} r_{\mathrm{exc}} R_{\mathrm{co}}$.

Note that the factor $\frac{\pi^{2}}{6}(=1.6)$, which accounts for the emission under the under-excitation regime, is moderate. Therefore, the uncertain contribution of these outer regions have little influence on the total emission. 
D. Teyssier et al.: CO line emission from circumstellar envelopes, Online Material $p 4$
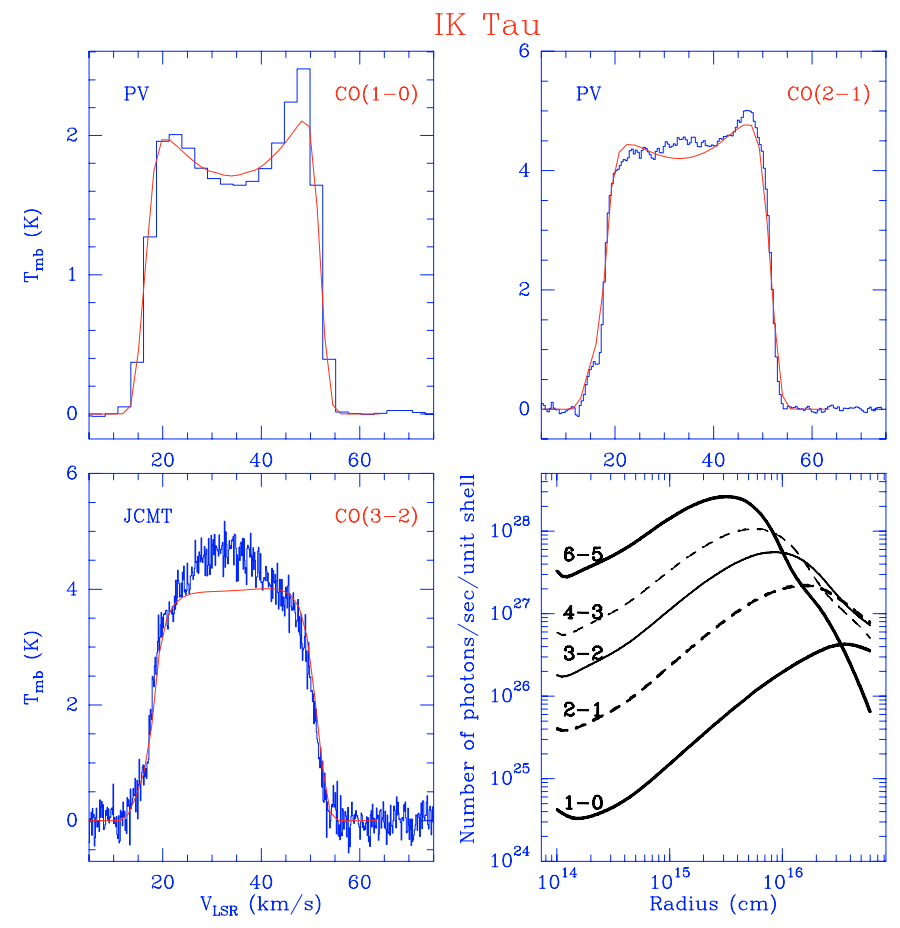

Fig. 4. Same as Fig. 2 for IK Tau. 
D. Teyssier et al.: CO line emission from circumstellar envelopes, Online Material p 5
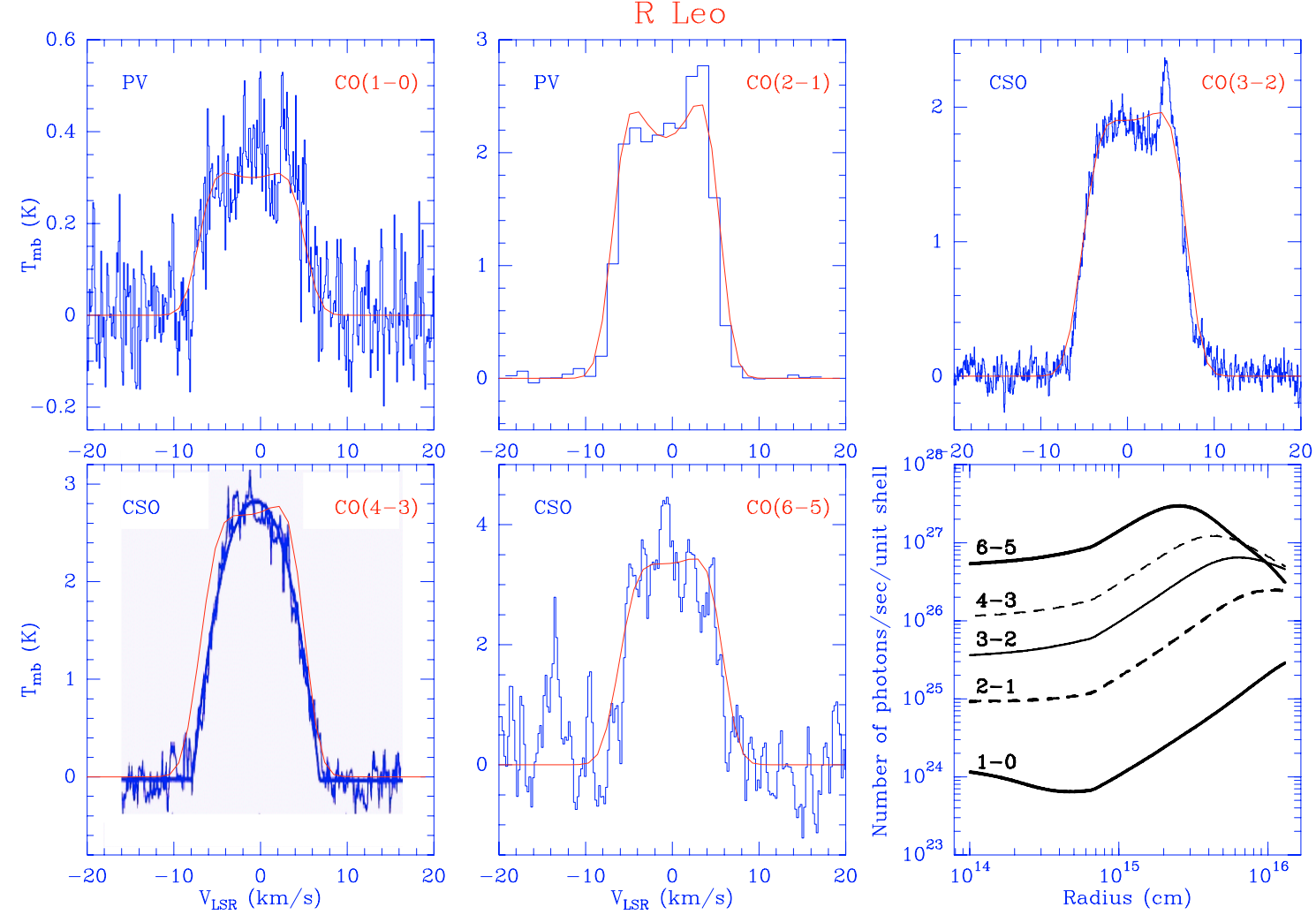

Fig. 5. Same as Fig. 2 for R Leo. The thick blue line following the $\operatorname{CO}(J=4-3)$ profile corresponds to the fit found by Young et al. (1995).
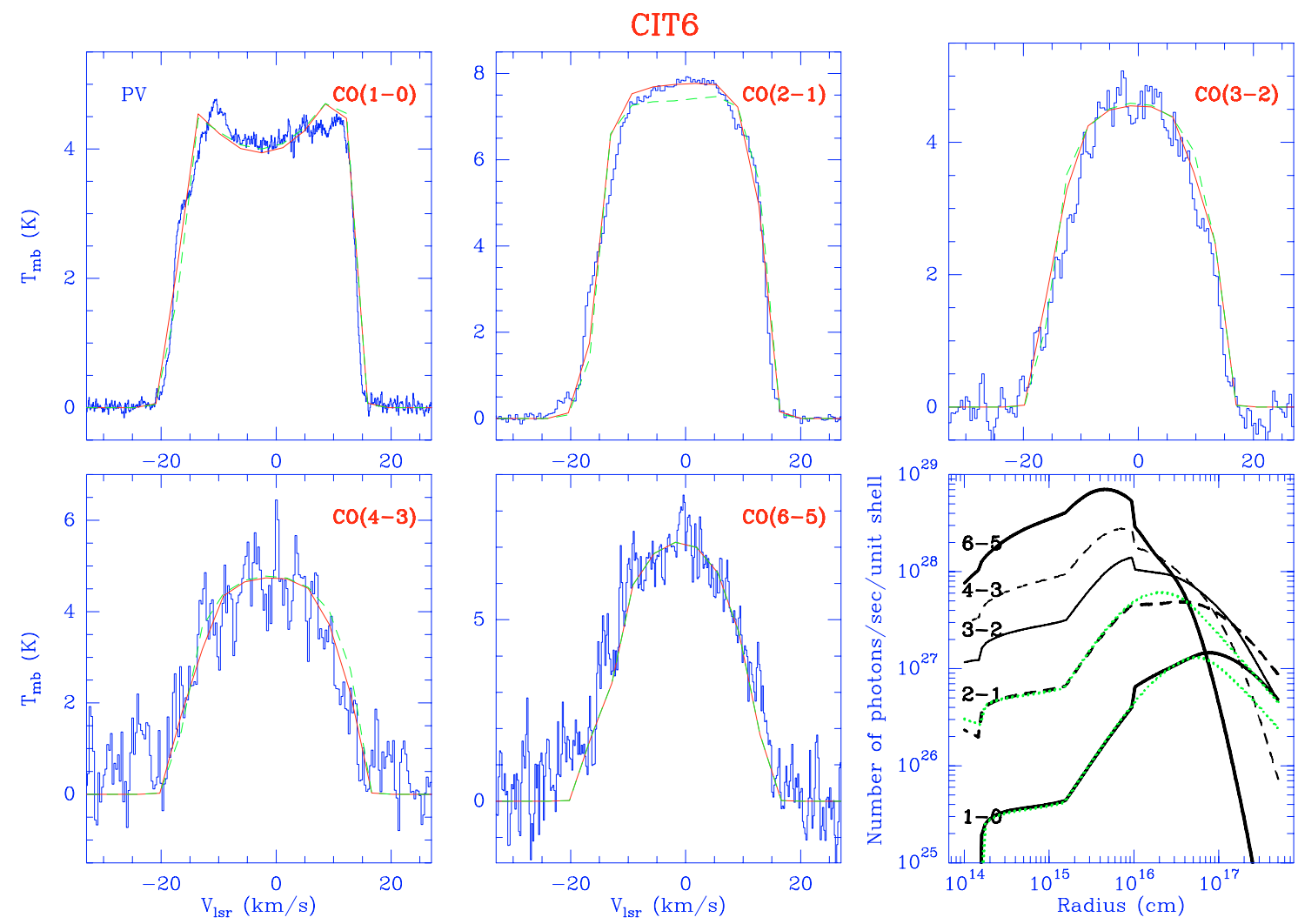

Fig. 6. Same as Fig. 2 for CIT 6. Here best-fit spectra are displayed for a single component model (dashed green lines) and for a double component model (full red lines). The number of photons is also shown for the 1-component model in the (1-0) and (2-1) transitions (Green dotted lines in the lower right panel). See text for details. 
D. Teyssier et al.: CO line emission from circumstellar envelopes, Online Material p 6
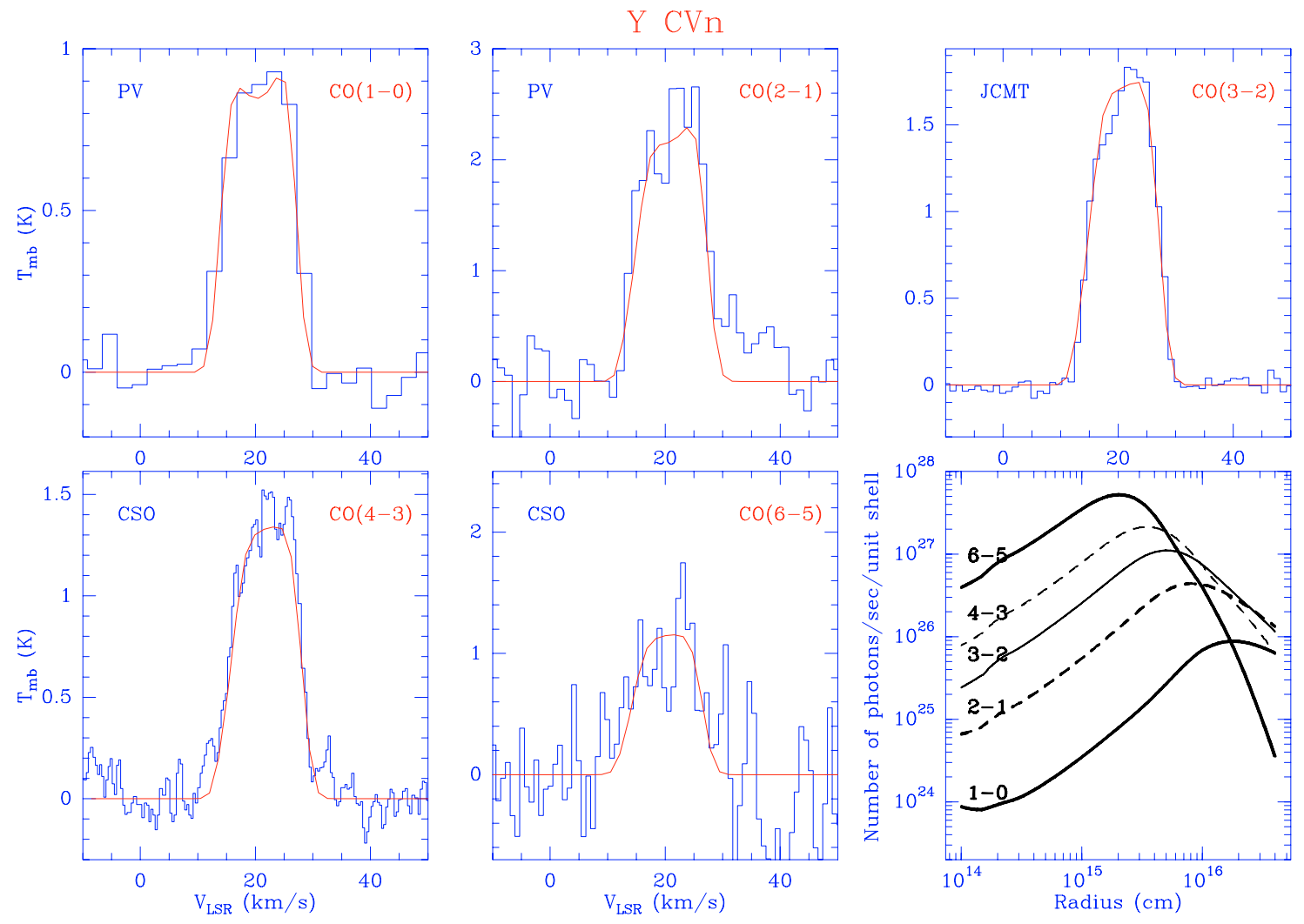

Fig. 7. Same as Fig. 2 for Y CVn.

R Hya
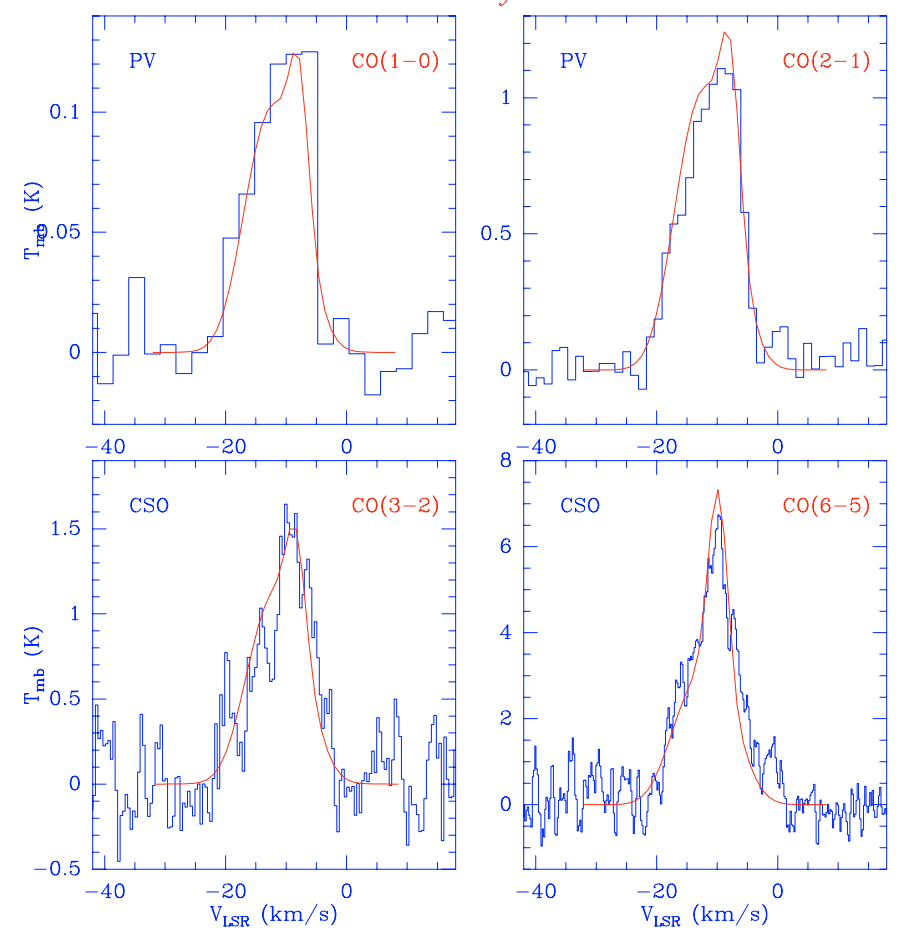

Fig. 8. Same as Fig. 2 for R Hya. 
D. Teyssier et al.: CO line emission from circumstellar envelopes, Online Material p 7
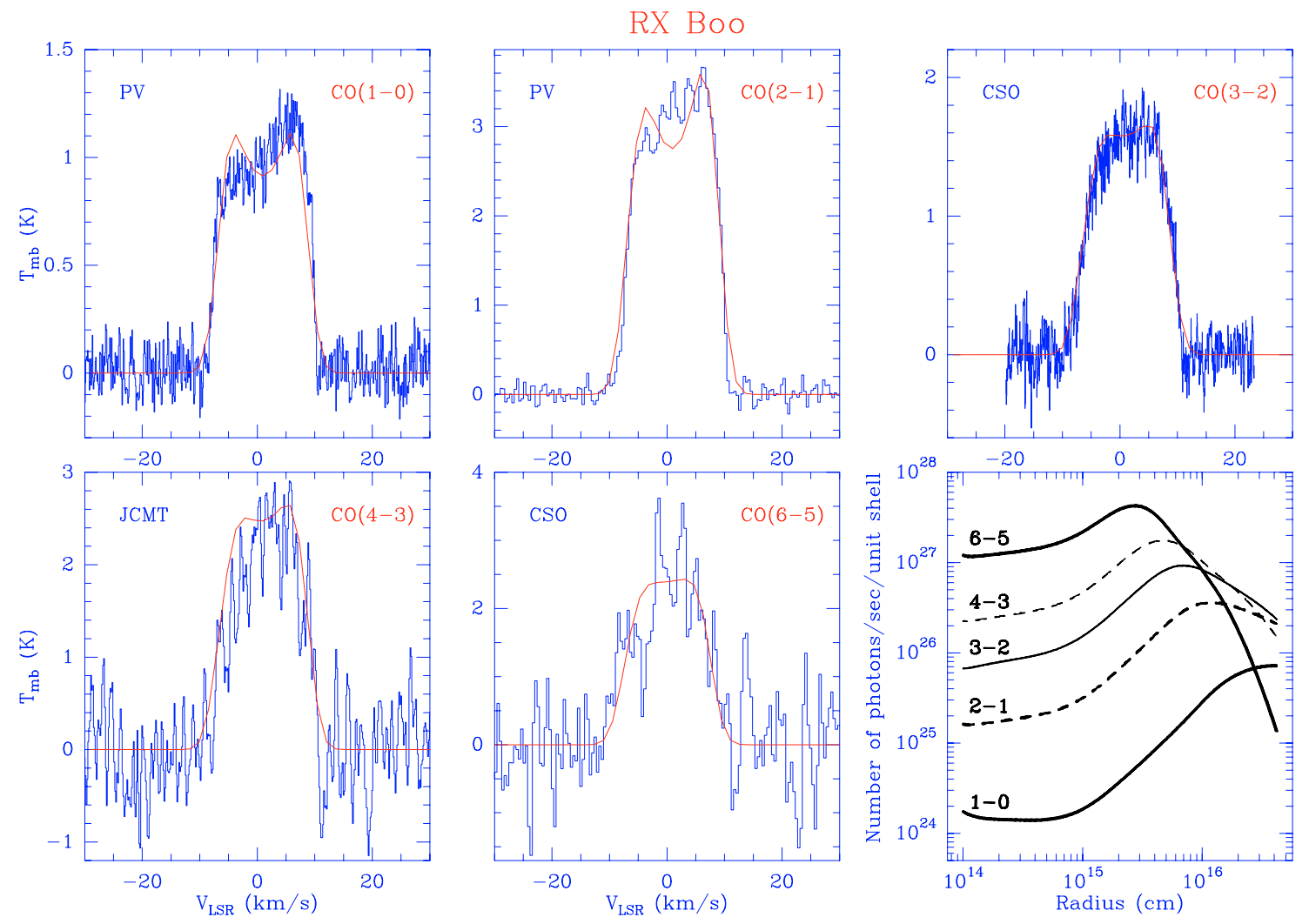

Fig. 9. Same as Fig. 2 for RX Boo.

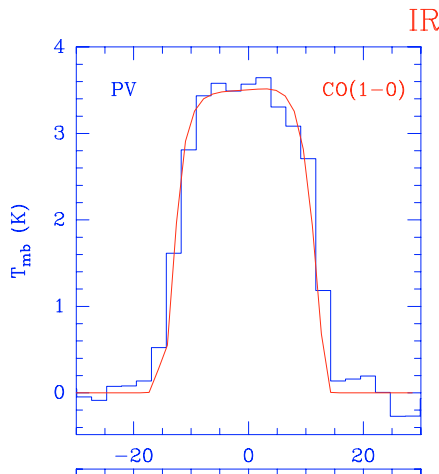

$\mathrm{IRC}+20370$
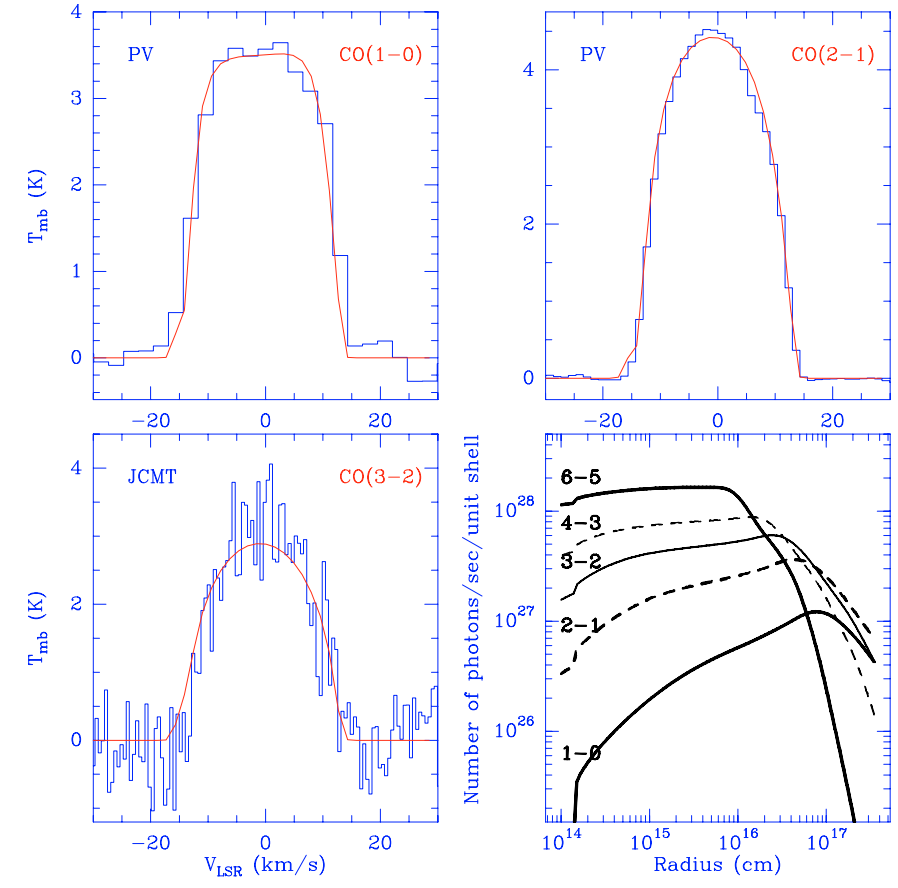

Fig. 10. Same as Fig. 2 for IRC +20370 . 
D. Teyssier et al.: CO line emission from circumstellar envelopes, Online Material p 8
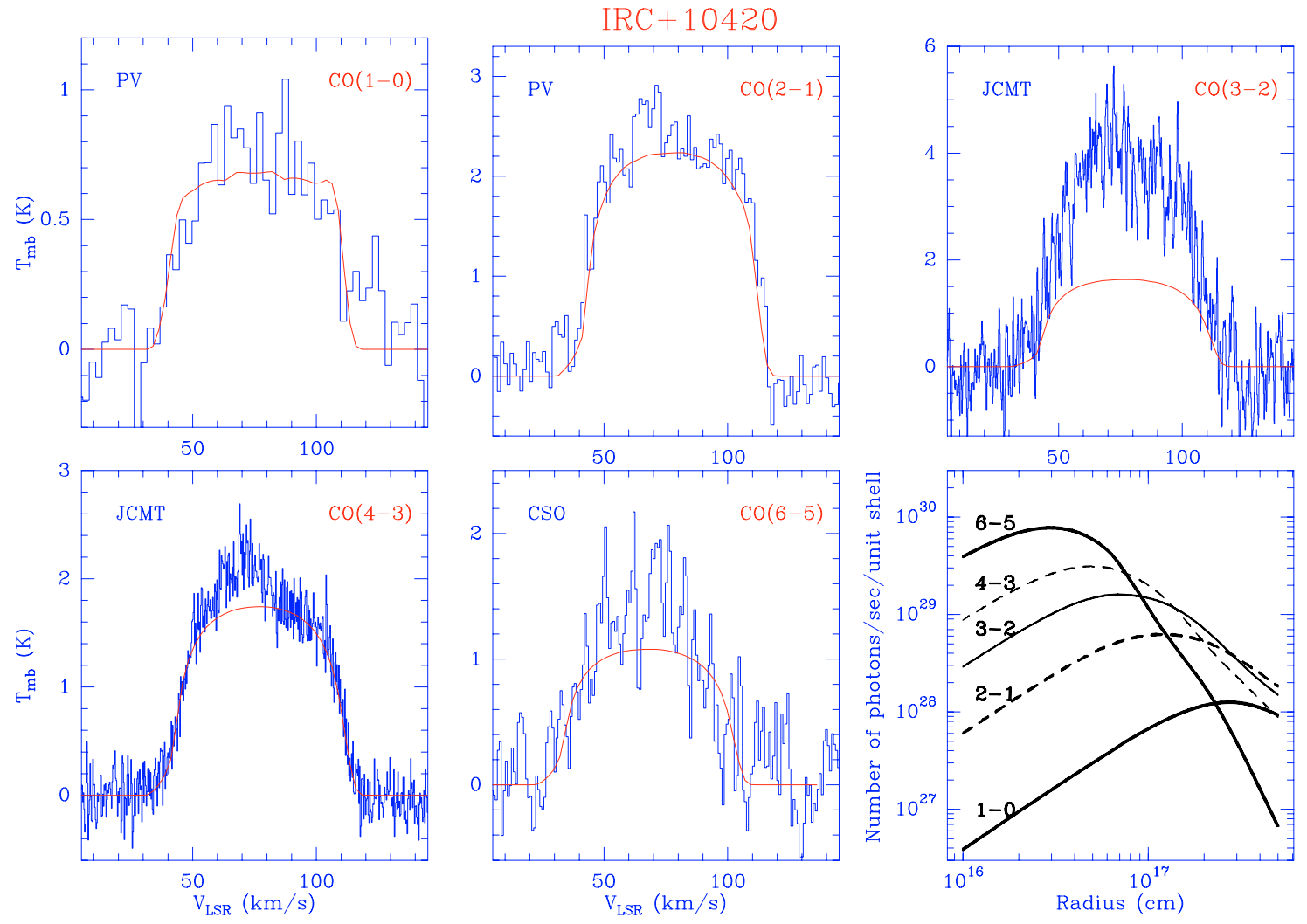

Fig. 11. Same as Fig. 2 for IRC+10420.
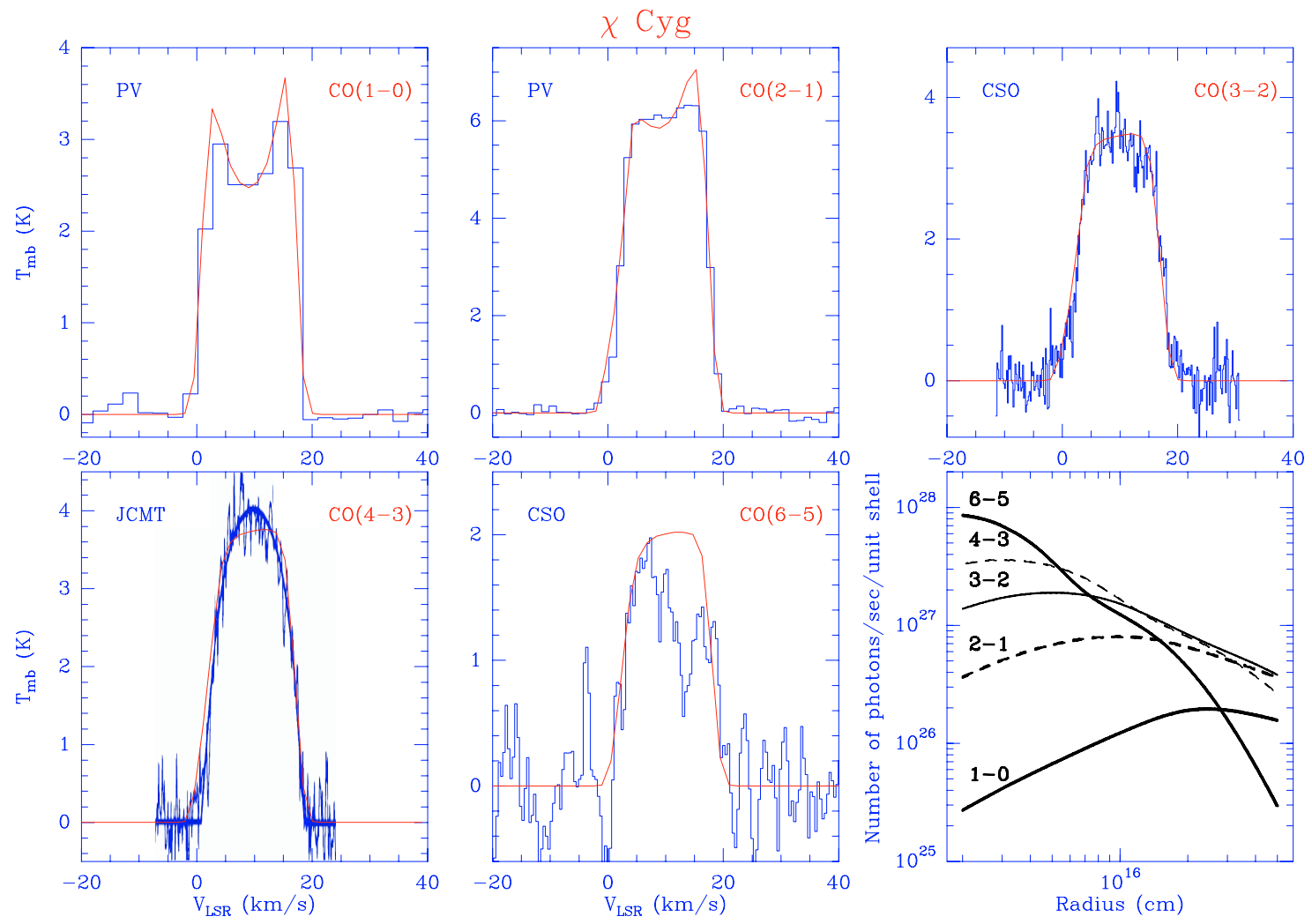

Fig. 12. Same as Fig. 2 for $\chi$ Cyg. Note that the $\operatorname{CO}(J=6-5)$ model is here shown with a calibration coefficient of 0.7 . The thick blue line following the $\mathrm{CO}(J=4-3)$ profile corresponds to the fit found by Young et al. (1995). 
D. Teyssier et al.: CO line emission from circumstellar envelopes, Online Material $p 9$
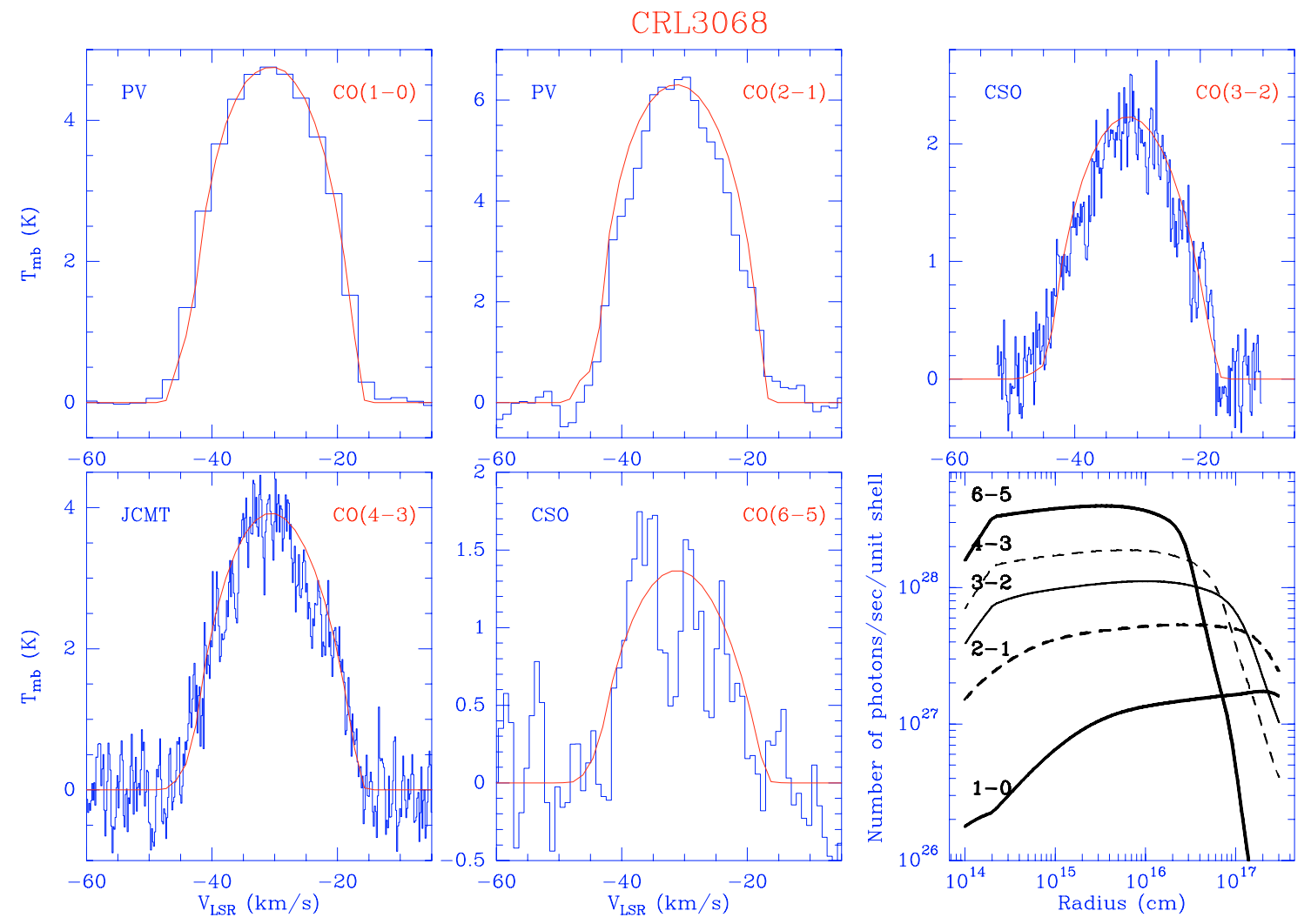

Fig. 13. Same as Fig. 2 for CRL 3068.
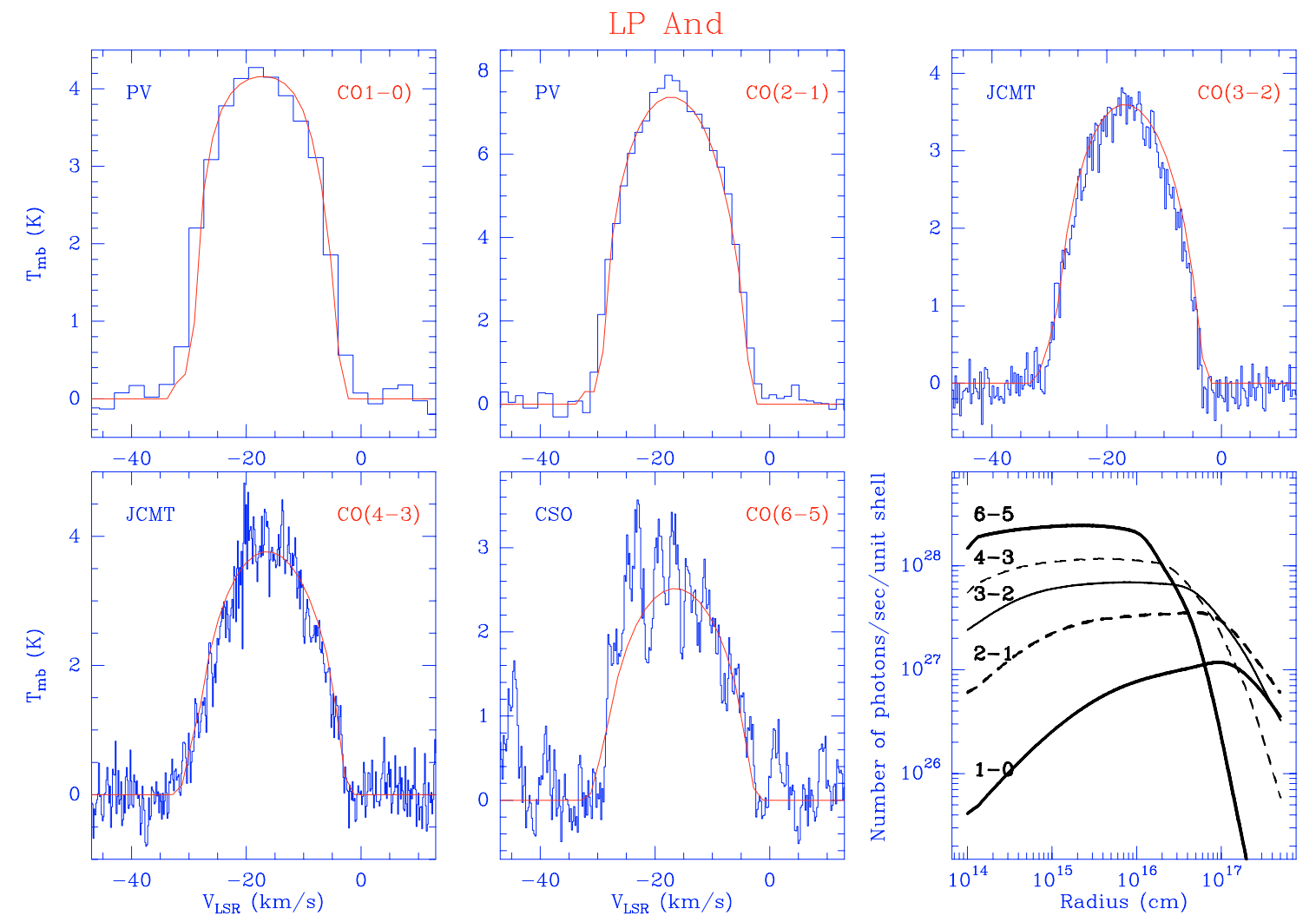

Fig. 14. Same as Fig. 2 for LP And. 
D. Teyssier et al.: CO line emission from circumstellar envelopes, Online Material p 10

IK Tau

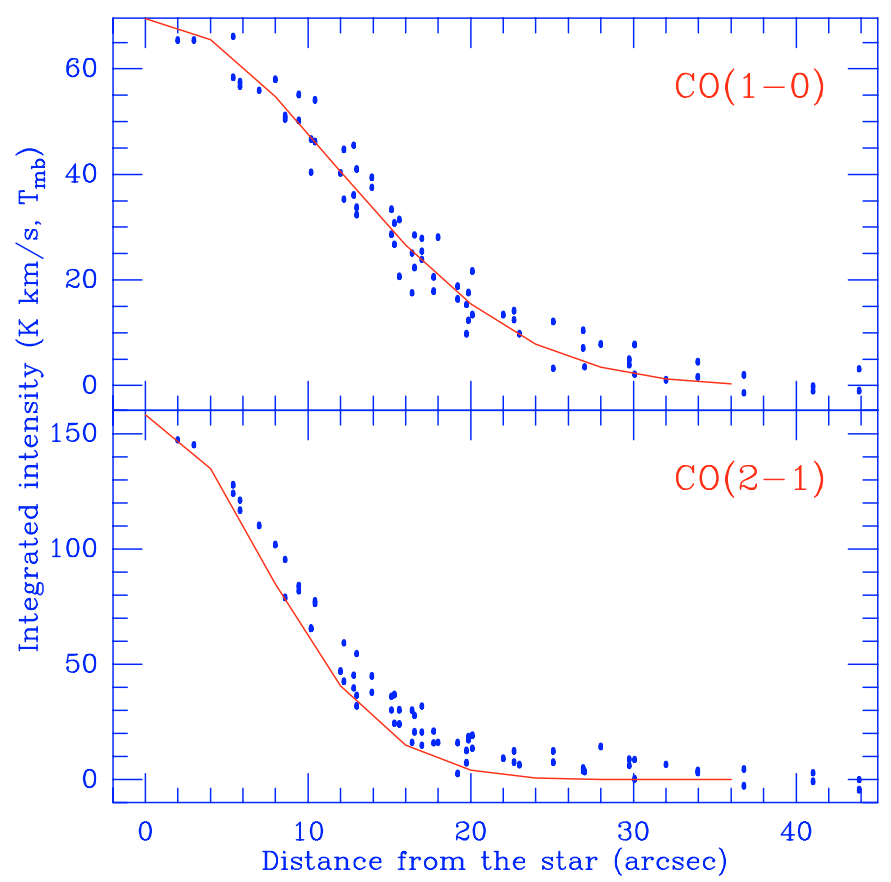

Fig. 15. Best fit model to the $\mathrm{CO}(1-0)$ and $\mathrm{CO}(2-1)$ integrated intensities as a function of angular offset to the star center. The data are taken from Neri et al. (1998) and are, when necessary, re-centered by the adequate offset.
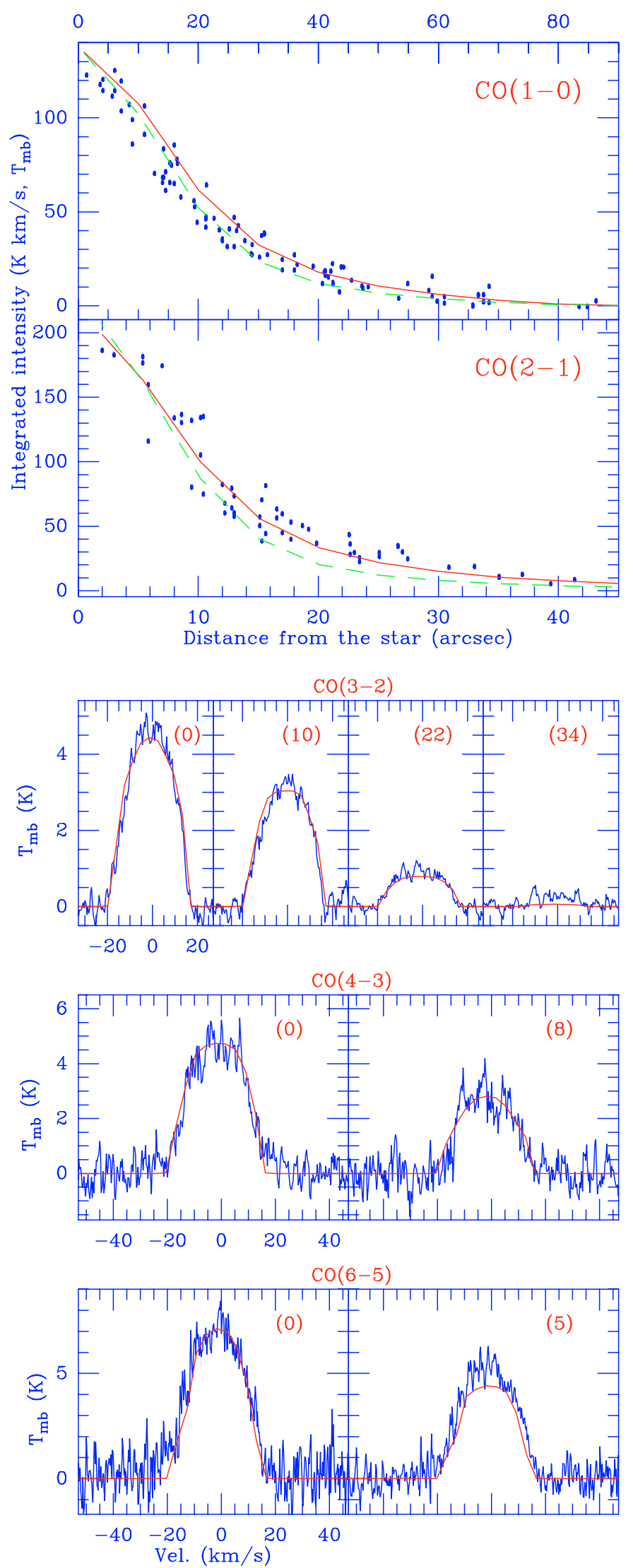

Fig. 16. Same as Fig. 3 for CIT 6. The radial cut are shown for a single component (green dashed line) and the two-component model (red full line) used to obtain the fit displayed in Fig. 6. 
D. Teyssier et al.: CO line emission from circumstellar envelopes, Online Material p 11
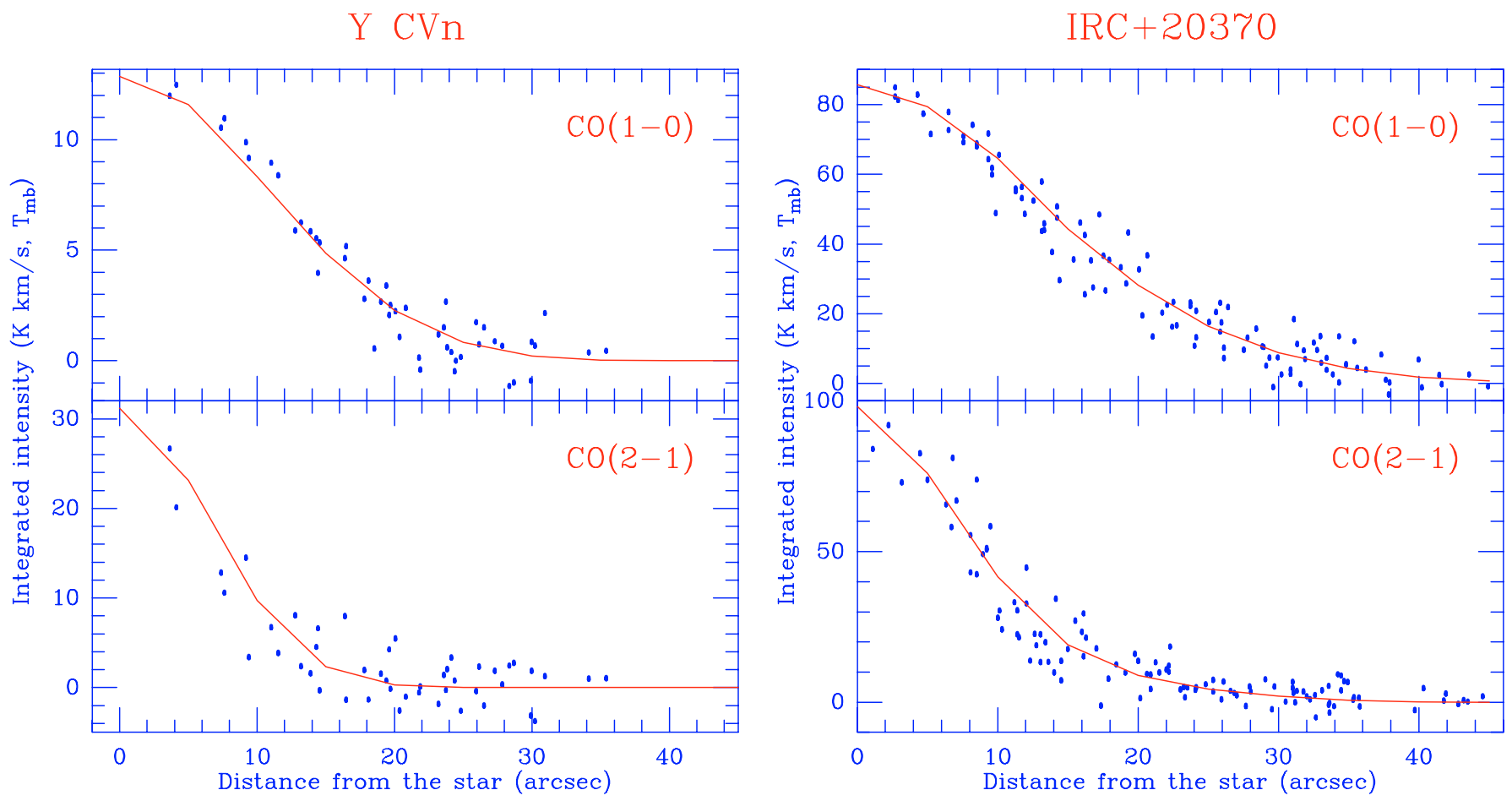

Fig. 17. Same as Fig. 15 for Y CVn.

Fig. 19. Same as Fig. 15 for IRC+20370.
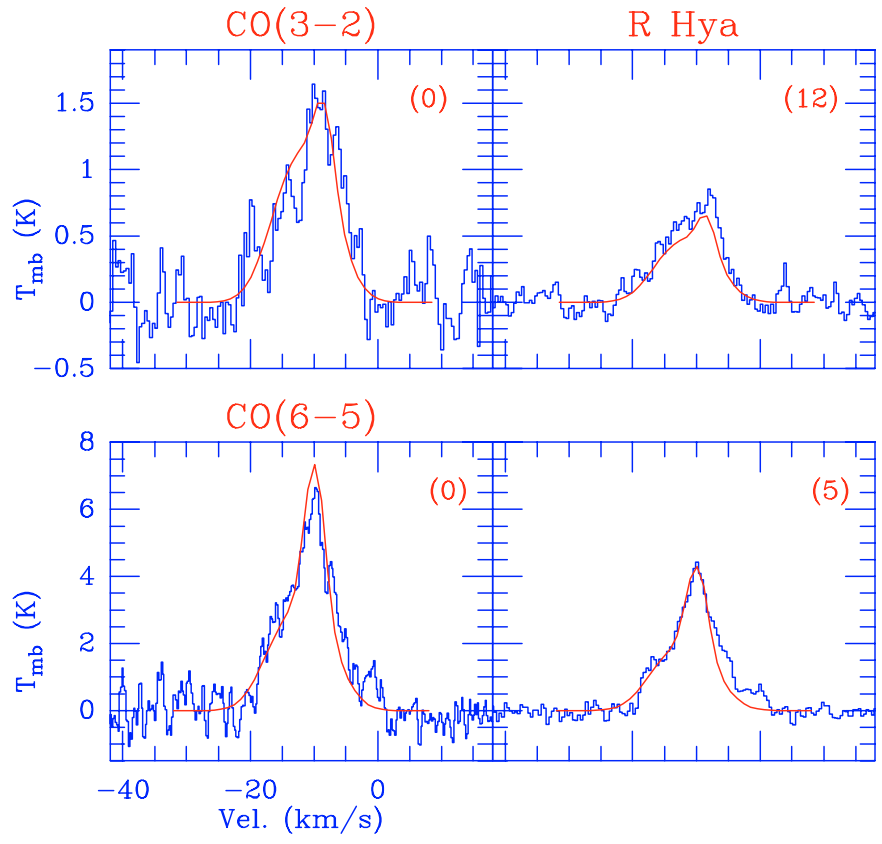

Fig. 18. Same as Fig. 3 for R Hya. Only two offsets per transitions could be observed here. 
D. Teyssier et al.: CO line emission from circumstellar envelopes, Online Material p 12

IRC +10420
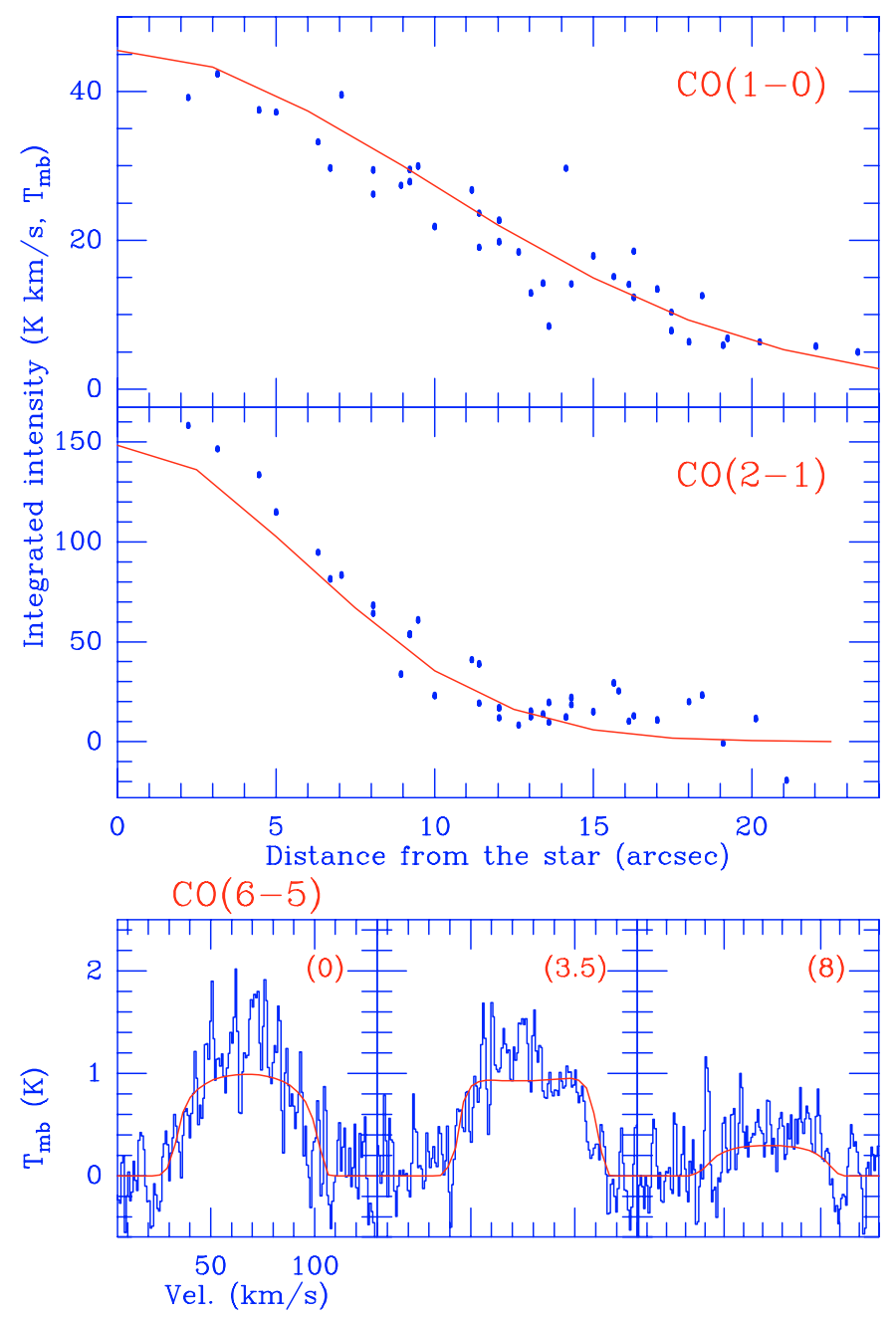

Fig. 20. Same as Fig. 3 for IRC+10420.

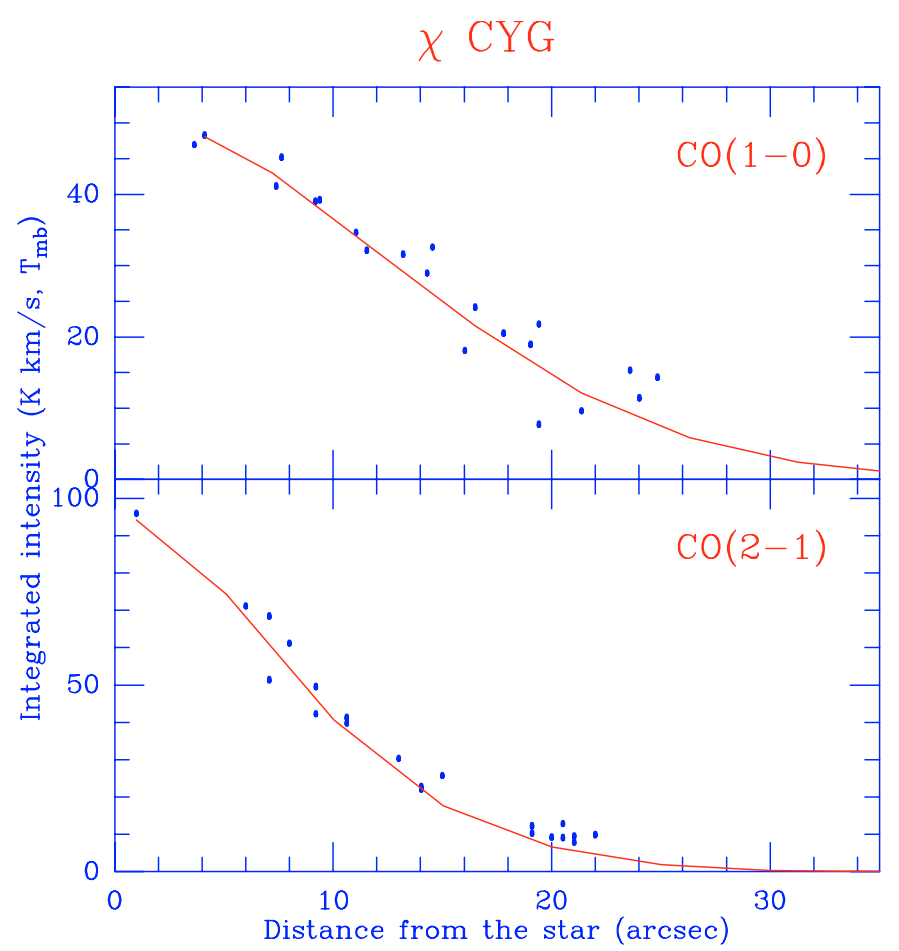

Fig. 21. Same as Fig. 15 for $\chi$ Cyg.

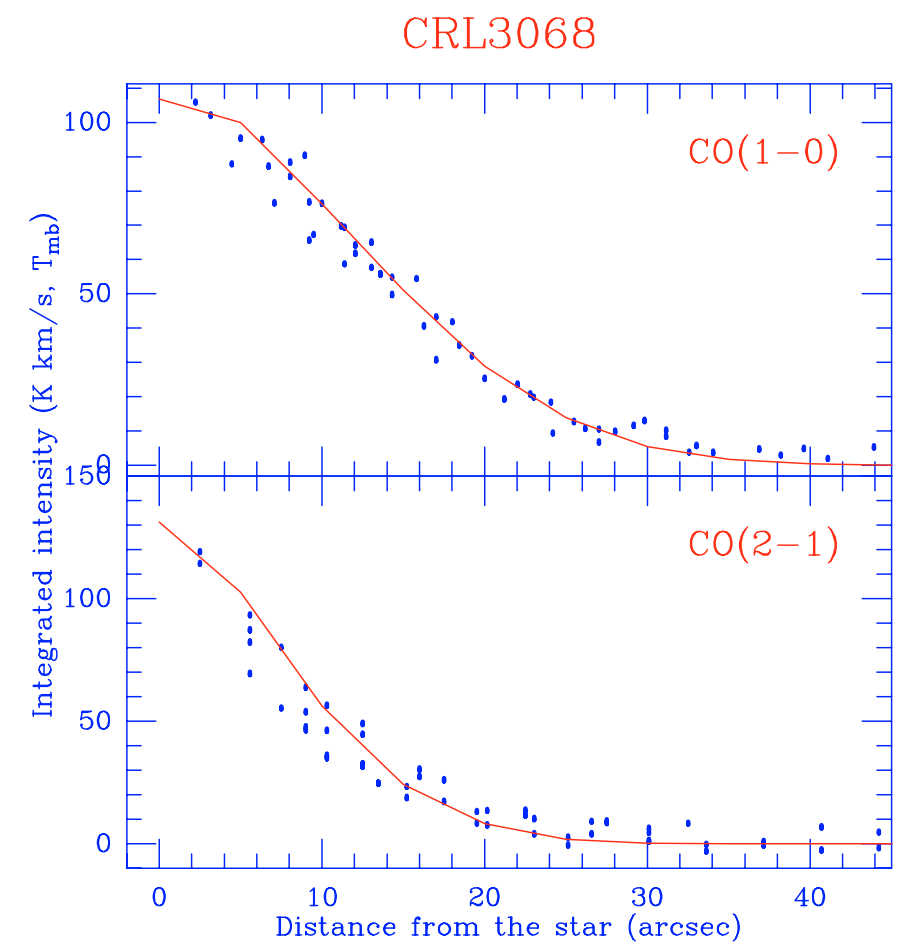

Fig. 22. Same as Fig. 15 for CRL 3068. 
D. Teyssier et al.: CO line emission from circumstellar envelopes, Online Material p 13
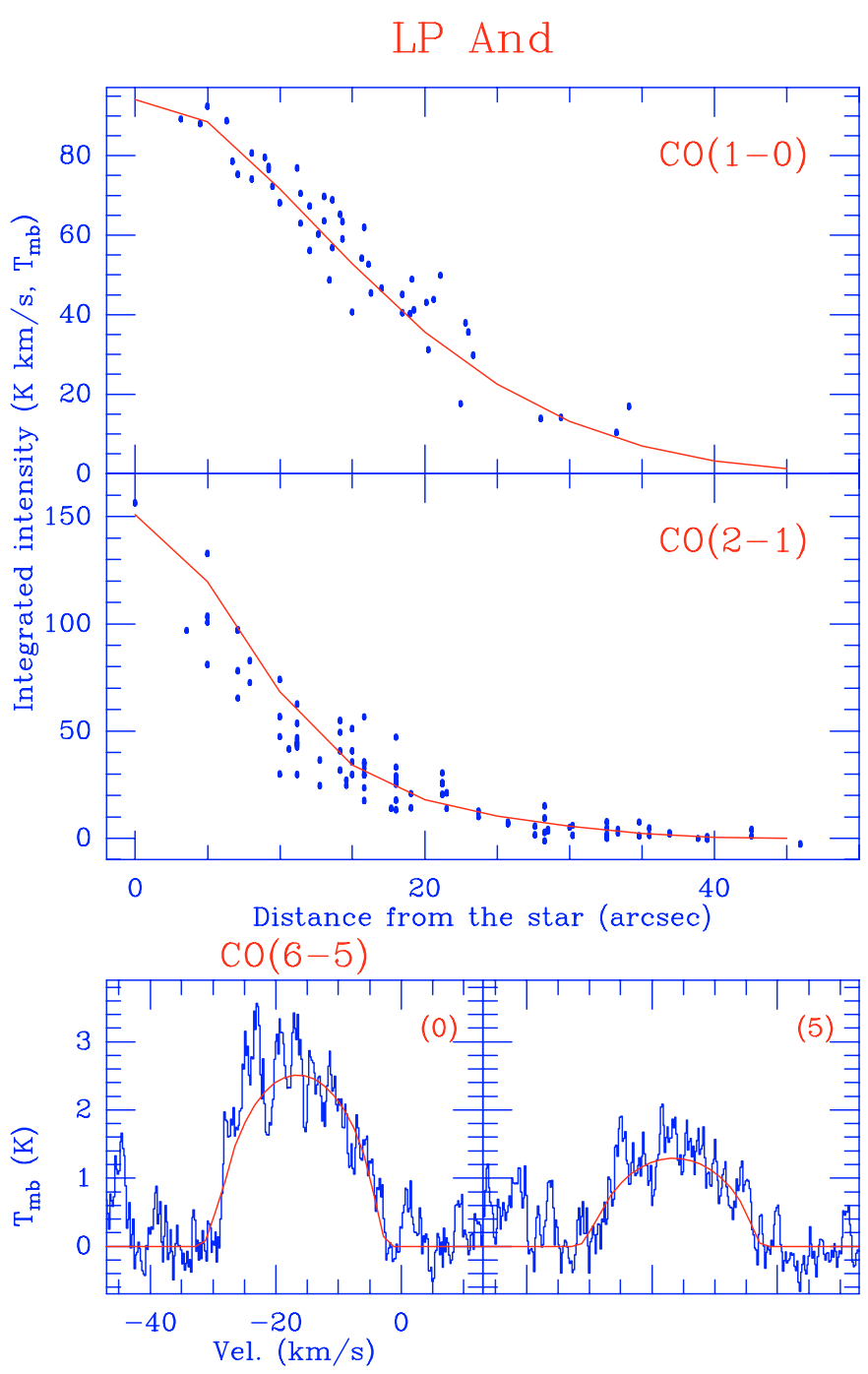

Fig. 23. Same as Fig. 3 for LP And. 